\title{
Closed-Form Solution to the Position Analysis of Watt-Baranov Trusses Using the Bilateration Method
}

\author{
Nicolás Rojas* and Federico Thomas \\ Institut de Robòtica i Informàtica Industrial (CSIC-UPC) \\ Llorens Artigas 4-6, 08028 Barcelona, Spain \\ \{nrojas, fthomas\}@iri.upc.edu
}

\begin{abstract}
The exact position analysis of a planar mechanism reduces to compute the roots of its characteristic polynomial. Obtaining this polynomial almost invariably involves, as a first step, obtaining a system of equations derived from the independent kinematic loops of the mechanism. The use of kinematic loops to this end has seldom been questioned despite deriving the characteristic polynomial from them requires complex variable eliminations and, in most cases, trigonometric substitutions. As an alternative, the bilateration method has recently been used to obtain the characteristic polynomials of the three-loop Baranov trusses without relying on variable eliminations nor trigonometric substitutions, and using no other tools than elementary algebra. This paper shows how this technique can be applied to members of a family of Baranov trusses resulting from the circular concatenation of the Watt mechanism irrespective of the resulting number of kinematic loops. To our knowledge, this is the first time that the characteristic polynomial of a Baranov truss with more that five loops has been obtained and, hence, its position analysis solved in closed form.
\end{abstract}

Keywords: Baranov trusses, Assur kinematic chains, position analysis, bilateration, distance-based formulations.

\section{Introduction}

The position analysis of planar linkages has been dominated by resultant elimination and tangent-half-angle substitution techniques applied to sets of kinematic loop equations. This analysis is thus reduced to finding the roots of a polynomial in one variable, the characteristic polynomial of the linkage. When this polynomial is obtained, it is said that the problem is solved in closed form. This approach is usually preferred to numerical approaches because the degree of the polynomial specifies the greatest possible number of assembly configurations of the linkage and modern software of personal computers provides guaranteed and fast computation of all real roots of a polynomial equation and hence of all assembly configurations of the analyzed linkage.

A non-overconstrained linkage with zero-mobility from which an Assur group can be obtained by removing any of its links is defined as an Assur kinematic chain, basic truss [1,2], or Baranov ${ }^{1}$ truss when no slider joints are considered [3]. Hence, a Baranov truss, named after the Russian kinematician G.G. Baranov [4] who first stated it in 1952 [5], corresponds to multiple Assur groups. The relevance of the Baranov trusses derive from the fact that, if the position analysis of a Baranov truss is solved, the same process can be applied to solve the position analysis of all its corresponding Assur groups. Curiously enough, despite this importance, it is commonly accepted that the Baranov trusses with more than 9 links have not been properly catalogued yet while all Assur groups with up to 12 links have been identified (see Table 1) [3]. It is worth mentioning here that Yang and Yao found that the number of Baranov trusses with 11 links is 239 using an algorithm that certainly requires further attention [6].

While the standard closed-form position analysis leads to complex systems of non-linear equations derived from independent kinematic loop equations, the bilateration method avoids the computation of loop equations as usually understood. It has recently been shown to be a powerful technique by obtaining the characteristic polynomial of the three 3-loop Baranov trusses without relying on variable eliminations nor half-angle tangent substitutions [7].

At the end of the XIX century, it was known that there were only two six-link single-dof planar hinged linkages. At a suggestion of Burmester [8], these two linkages were called the Watt linkage and the Stephenson linkage. Several Stephenson linkages can be concatenated leading to what in [9] was called a Stephenson pattern. Likewise, several Watt linkages can be concatenated to obtain what can be called, for the same reason, a Watt pattern (see [10] for their motion

\footnotetext{
* Address all correspondence to this author.

${ }^{1}$ Some authors misspell it as Barranov.
} 
simulations). If these concatenations are circular, the results are Baranov trusses which will be called Stepheson-Baranov and Watt-Baranov trusses, respectively (Fig. 1).

Table 1. Number of Baranov trusses as a function of the number of links (alternatively, number of loops), and number of different Assur groups resulting from eliminating one link from the Baranov trusses in each class $[3,6]$.

\begin{tabular}{cccc}
\hline Links & Loops & $\begin{array}{c}\text { Baranov } \\
\text { trusses }\end{array}$ & $\begin{array}{c}\text { Resulting } \\
\text { Assur groups }\end{array}$ \\
\hline 3 & 1 & 1 & 1 \\
5 & 2 & 1 & 2 \\
7 & 3 & 3 & 10 \\
9 & 4 & 28 & 173 \\
11 & 5 & 239 & 5442 \\
13 & 6 & unknown & 251638 \\
\hline
\end{tabular}

The position analysis of the Stepheson-Baranov truss of 4 loops has been solved in closed form at least in [11-14], and more recently by $\mathrm{K}$. Wohlhart in [15] thus reaching what the author considers to be the limit of Sylvester's elimination method. The position analysis of the Watt-Baranov truss of 4 loops was solved in closed form by L. Han et. al. in [16] and more recently by J. Borràs and R. Di Gregorio [17]. Elimination methods seem to reach their limit with the analysis of Baranov trusses with four, or five loops, depending on their topology. Actually, the closed-form position analysis of a Baranov truss with more than five loops has not been reported to the best of our knowledge, and only the closed-form position analysis of one five-loop Baranov truss has been obtained $[12,18]$. In this paper, we address this challenge and we push the loop limit further by solving the closed-form position analysis of Watt-Baranov trusses, with up to six loops, using the bilateration method.

This paper is organized as follows. In Section 2, the basic formula required to apply the bilateration method is briefly reviewed. Then, in section 3 , it is shown how the bilateration method can be applied to obtain the characteristic polynomial of a Watt-Baranov truss with an arbitrary number of kinematic loops. To this end, it is first shown how to derive a single scalar radical equation which is satisfied if, an only if, the truss can be assemble and, then, how the characteristic polynomial is derived by simply clearing radicals. This last step is actually the only costly step in the whole process. Two examples are analyzed in Section 4, including a 6-loop WattBaranov truss -whose characteristic polynomial is of degree 126- with 76 assembly modes.

\section{Bilateration}

The bilateration problem consists of finding the feasible locations of a point, say $P_{k}$, given its distances to two other points, say $P_{i}$ and $P_{j}$, whose locations are known. Then, according to Fig. 2, the result, in matrix form, can be expressed as:

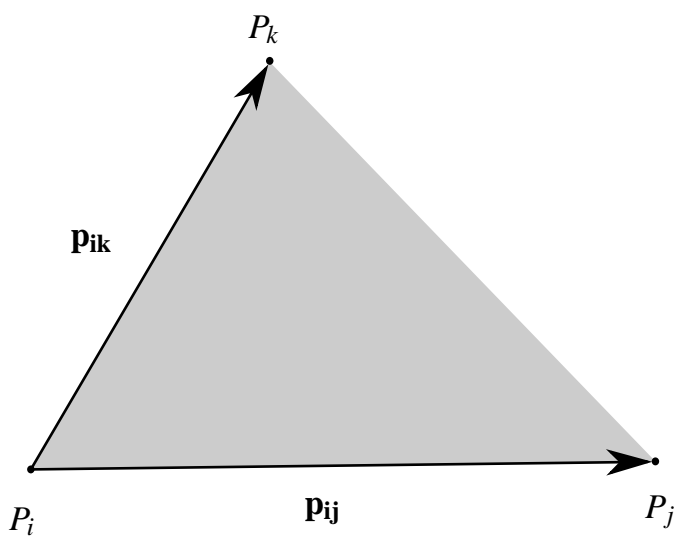

Fig. 2. The bilateration problem in $\mathbb{R}^{2}$.

$$
\mathbf{p}_{i k}=\mathbf{Z}_{i, j, k} \mathbf{p}_{i j}
$$

where

$$
\mathbf{Z}_{i, j, k}=\frac{1}{D(i, j)}\left[\begin{array}{cc}
D(i, j ; i, k) & \mp \sqrt{D(i, j, k)} \\
\pm \sqrt{D(i, j, k)} & D(i, j ; i, k)
\end{array}\right],
$$

is called a bilateration matrix, and

$$
D\left(i_{1}, \ldots, i_{n} ; j_{1}, \ldots, j_{n}\right)=2\left(\frac{-1}{2}\right)^{n}\left|\begin{array}{cccc}
0 & 1 & \ldots & 1 \\
1 & s_{i_{1}, j_{1}} & \ldots & s_{i_{1}, j_{n}} \\
\vdots & \vdots & \ddots & \vdots \\
1 & s_{i_{n}, j_{1}} & \ldots & s_{i_{n}, j_{n}}
\end{array}\right|
$$

with $s_{i, j}=d_{i, j}^{2}=\left\|\mathbf{p}_{i j}\right\|^{2}$, where $\mathbf{p}_{i j}=\mathbf{p}_{j}-\mathbf{p}_{i}=\vec{P}_{i} P_{j}$. This determinant is known as the Cayley-Menger bi-determinant of the point sequences $P_{i_{1}}, \ldots, P_{i_{n}}$, and $P_{j_{1}}, \ldots, P_{j_{n}}$ and its geometric interpretation plays a fundamental role in the so-called Distance Geometry, the analytical study of Euclidean geometry in terms of invariants [19]. When the two point sequences are the same, it is convenient to abbreviate $D\left(i_{1}, \ldots, i_{n} ; i_{1}, \ldots, i_{n}\right)$ by $D\left(i_{1}, \ldots, i_{n}\right)$, which is simply called the Cayley-Menger determinant of the involved points.

Now, it is important to observe that this kind of matrices constitute an Abelian group under product and addition and if $\mathbf{v}=\mathbf{Z w}$, where $\mathbf{Z}$ is a bilateration matrix, then $\|\mathbf{v}\|^{2}=\operatorname{det}(\mathbf{Z})\|\mathbf{w}\|^{2}$. The interested reader is addressed to [7] for a more detailed treatment of bilateration matrices and some basic geometric operations that can be performed with them.

\section{Position Analysis of the General N-link Watt-Baranov Truss}

Fig. 3 shows the general $n$-link Watt-Baranov truss, a structure with $k=\frac{n-1}{2}$ loops and $v=\frac{3}{2}(n-1)$ revolute joints. The $k$-ary link is defined by $P_{1} P_{4} P_{7} \ldots P_{v-5} P_{v-2}$, and the 

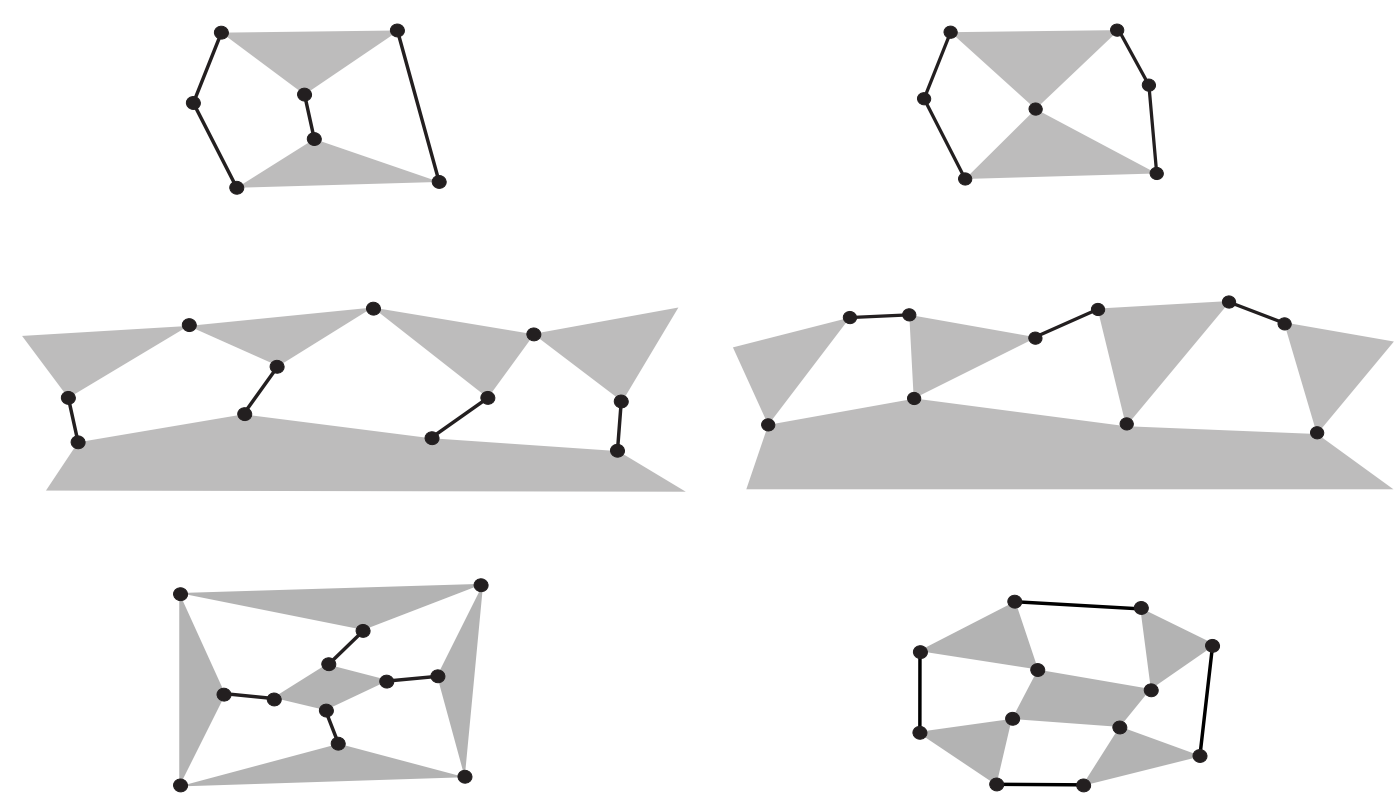

Fig. 1. Left column: The Stephenson linkage, the Stephenson pattern resulting from concatenating four Stephenson linkages, and the Stephenson-Baranov truss resulting form the circular concatenation of four Stephenson linkages. Right colum: The Watt linkage, the Watt pattern resulting from concatenating four Watt linkages, and the Watt-Baranov truss resulting form the circular concatenation of four Watt linkages.

$k$ ternary links by the triangles $P_{1} P_{v} P_{2}, P_{4} P_{3} P_{5}, P_{7} P_{6} P_{8}, \ldots$, $P_{v-5} P_{v-6} P_{v-4}$ and $P_{v-2} P_{v-3} P_{v-1}$. The position analysis problem for this structure consists in, given the dimensions of all links, calculating all relative possible transformations between them all. To solve this problem, instead of directly computing the relative Cartesian poses of all links through loop-closure equations, we will compute the set of values of $s_{1,3}$ compatible with all binary and ternary links side lengths. Thus, this procedure is entirely posed in terms of distances.

On the one hand, according to Fig. $3, \mathbf{p}_{1,4}, \mathbf{p}_{1,7}, \ldots$, $\mathbf{p}_{1, v-5}, \mathbf{p}_{1, v-2}$ can be expressed as a function of $\mathbf{p}_{1,3}$ using bilaterations as follows:

$$
\begin{aligned}
\mathbf{p}_{1,4} & =\mathbf{Z}_{1,3,4} \mathbf{p}_{1,3} \\
\mathbf{p}_{1,7} & =\mathbf{Z}_{1,4,7} \mathbf{p}_{1,4}=\mathbf{Z}_{1,4,7} \mathbf{Z}_{1,3,4} \mathbf{p}_{1,3} \\
\mathbf{p}_{1,10} & =\mathbf{Z}_{1,7,10} \mathbf{p}_{1,7}=\mathbf{Z}_{1,7,10} \mathbf{Z}_{1,4,7}, \mathbf{Z}_{1,3,4} \mathbf{p}_{1,3} \\
\vdots & \\
\mathbf{p}_{1, v-5} & =\mathbf{Z}_{1, v-8, v-5} \mathbf{Z}_{1, v-11, v-8} \ldots \mathbf{Z}_{1,4,7}, \mathbf{Z}_{1,3,4} \mathbf{p}_{1,3} \\
\mathbf{p}_{1, v-2} & =\mathbf{Z}_{1, v-5, v-2} \mathbf{Z}_{1, v-8, v-5} \ldots \mathbf{Z}_{1,4,7}, \mathbf{Z}_{1,3,4} \mathbf{p}_{1,3} .
\end{aligned}
$$

On the other hand, for the ternary link $P_{4} P_{3} P_{5}$, we have

$$
\begin{aligned}
\mathbf{p}_{4,5} & =\mathbf{Z}_{4,3,5} \mathbf{p}_{4,3} \\
\mathbf{p}_{4,1}+\mathbf{p}_{1,5} & =\mathbf{Z}_{4,3,5}\left(\mathbf{p}_{4,1}+\mathbf{p}_{1,3}\right) \\
\mathbf{p}_{1,5} & =\mathbf{p}_{1,4}+\mathbf{Z}_{4,3,5}\left(\mathbf{p}_{1,3}-\mathbf{p}_{1,4}\right) .
\end{aligned}
$$

Likewise, for the ternary links $P_{7} P_{6} P_{8}, \ldots, P_{v-5} P_{v-6} P_{v-4}$ and
$P_{v-2} P_{v-3} P_{v-1}$, we obtain

$$
\begin{aligned}
& \mathbf{p}_{1,6}=\mathbf{p}_{1,7}+\mathbf{Z}_{7,5,6}\left(\mathbf{p}_{1,5}-\mathbf{p}_{1,7}\right) \\
& \mathbf{p}_{1,8}=\mathbf{p}_{1,7}+\mathbf{Z}_{7,6,8}\left(\mathbf{p}_{1,6}-\mathbf{p}_{1,7}\right) \\
& \vdots \\
& \mathbf{p}_{1, v-3}=\mathbf{p}_{1, v-2}+\mathbf{Z}_{v-2, v-4, v-3}\left(\mathbf{p}_{1, v-4}-\mathbf{p}_{1, v-2}\right) \\
& \mathbf{p}_{1, v-1}=\mathbf{p}_{1, v-2}+\mathbf{Z}_{v-2, v-3, v-1}\left(\mathbf{p}_{1, v-3}-\mathbf{p}_{1, v-2}\right) .
\end{aligned}
$$

Now, substituting (4)-(8) in (9)-(13) and then replacing the resulting expression for $\mathbf{p}_{1,5}$ in that for $\mathbf{p}_{1,6}$, and the resulting expression for $\mathbf{p}_{1,6}$ after this substitution in that for $\mathbf{p}_{1,8}$, and so on till an expression is obtained for $\mathbf{p}_{1, v-1}$, we get

$$
\mathbf{p}_{1, v-1}=\mathbf{Q}_{n} \mathbf{p}_{1,3}
$$

Moreover, for the ternary link $P_{1} P_{v} P_{2}$, we have

$$
\mathbf{p}_{1, v}=\mathbf{Z}_{1,2, v} \mathbf{Z}_{1,3,2} \mathbf{p}_{1,3}
$$

Finally, using equations (14) and (15), we get

$$
\mathbf{p}_{v-1, v}=\mathbf{p}_{v-1,1}+\mathbf{p}_{1, v}=\left(-\mathbf{Q}_{n}+\mathbf{Z}_{1,2, v} \mathbf{Z}_{1,3,2}\right) \mathbf{p}_{1,3}
$$

Therefore,

$$
\operatorname{det}\left(-\mathbf{Q}_{n}+\mathbf{Z}_{1,2, v} \mathbf{Z}_{1,3,2}\right)=\frac{s_{v-1, v}}{s_{1,3}} .
$$




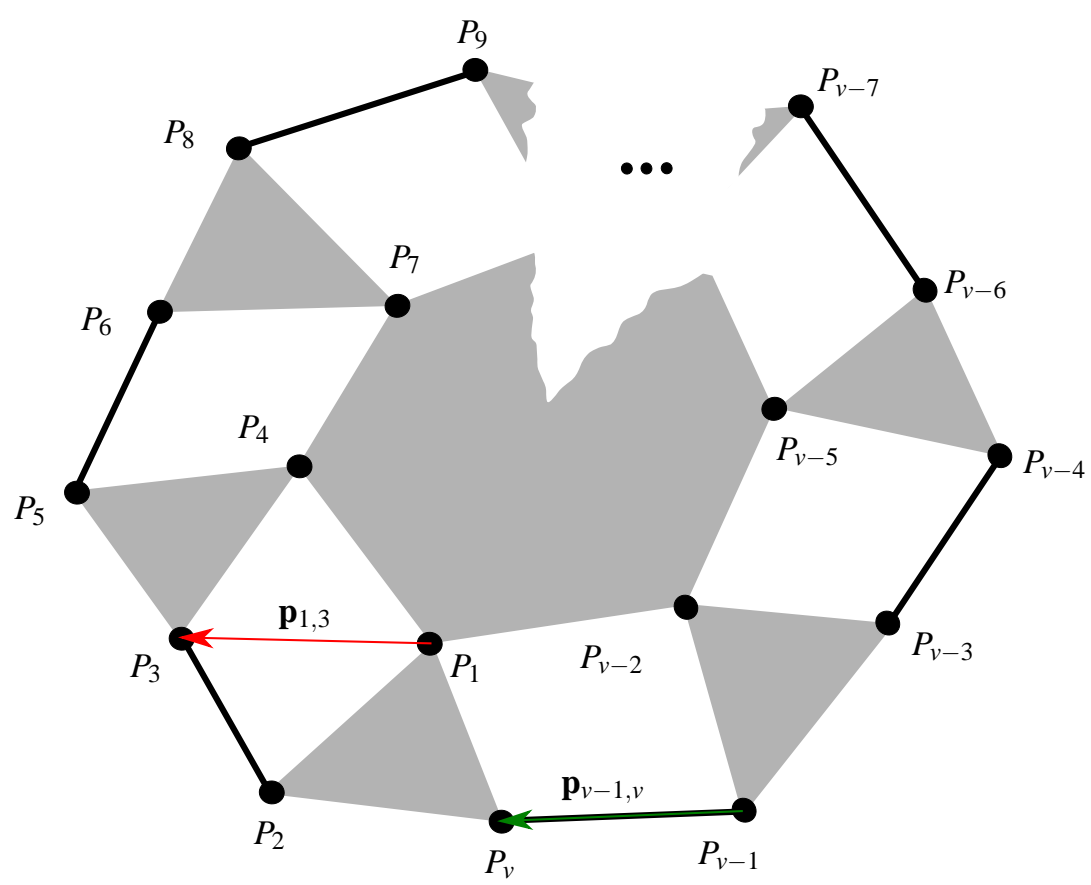

Fig. 3. The general $n$-link Watt-Baranov truss has $k=\frac{n-1}{2}$ loops and $v=\frac{3}{2}(n-1)$ revolute joints. $\mathbf{p}_{v-1, v}$ can be expressed as a function of $\mathbf{p}_{1,3}$ by computing $3 k-2$ bilaterations.

The left hand side of the above equation is a function of the $k-1$ unknown squared distances $s_{1,3}$ and $s_{5,7}, s_{8,10}, \ldots$, $s_{v-7, v-5}, s_{v-4, v-2}$.

Since, using the same procedure to obtain (16), allows us to obtain

$$
\begin{aligned}
& \mathbf{p}_{5,7}=-\mathbf{p}_{1,5}+\mathbf{p}_{1,7}=\mathbf{D}_{n_{1}} \mathbf{p}_{1,3} \\
& \mathbf{p}_{8,10}=-\mathbf{p}_{1,8}+\mathbf{p}_{1,10}=\mathbf{D}_{n_{2}} \mathbf{p}_{1,3} \\
& \vdots \\
& \mathbf{p}_{v-7, v-5}=-\mathbf{p}_{1, v-7}+\mathbf{p}_{1, v-5}=\mathbf{D}_{n_{k-3}} \mathbf{p}_{1,3} \\
& \mathbf{p}_{v-4, v-2}=-\mathbf{p}_{1, v-4}+\mathbf{p}_{1, v-2}=\mathbf{D}_{n_{k-2}} \mathbf{p}_{1,3} .
\end{aligned}
$$

Therefore,

$$
\begin{aligned}
s_{5,7} & =\operatorname{det}\left(\mathbf{D}_{n_{1}}\right) s_{1,3} \\
s_{8,10} & =\operatorname{det}\left(\mathbf{D}_{n_{2}}\right) s_{1,3} \\
\vdots & \\
s_{v-7, v-5} & =\operatorname{det}\left(\mathbf{D}_{n_{k-3}}\right) s_{1,3} \\
s_{v-4, v-2} & =\operatorname{det}\left(\mathbf{D}_{n_{k-2}}\right) s_{1,3} .
\end{aligned}
$$

The substitution of (22) - (25) into (17) yields a scalar equation in a single variable: $s_{1,3}$. The roots of this equation, in the range in which the signed areas of the triangles $P_{1} P_{2} P_{3}$ and $P_{1} P_{3} P_{4}$ are real, that is, the range

$$
\begin{aligned}
& {\left[\max \left\{\left(d_{1,2}-d_{2,3}\right)^{2},\left(d_{1,4}-d_{3,4}\right)^{2}\right\},\right.} \\
& \left.\min \left\{\left(d_{1,2}+d_{2,3}\right)^{2},\left(d_{1,4}+d_{3,4}\right)^{2}\right\}\right]
\end{aligned}
$$

determine the assembly modes of the general $n$-link WattBaranov truss. These roots can be readily obtained using, for example, an interval Newton method for the $2^{k}$ possible combinations for the signs of the signed areas of the triangles $P_{1} P_{2} P_{3}, P_{1} P_{3} P_{4}$, and $P_{7} P_{5} P_{6}, P_{10} P_{8} P_{9}, \ldots, P_{v-5} P_{v-7} P_{v-6}$, $P_{v-2} P_{v-4} P_{v-3}$.

In order to obtain the characteristic polynomial it just remains to clear all square roots in the obtained scalar equation by isolating one at a time and squaring the result till no square root remains. Using a computer algebra system, it can be seen that this clearing process leads to

$$
s_{1,3}^{2^{k-1}} s_{5,7}^{2^{k-2}} s_{8,10}^{2^{k-3}} \ldots s_{v-7, v-5}^{4} s_{v-4, v-2}^{2} \Delta_{n}=0
$$

where $\Delta_{n}$ is a polynomial in $s_{1,3}$ of degree $2^{k+1}-2$. The extraneous roots at $s_{1,3}=0, \ldots, s_{v-4, v-2}=0$ were introduced when clearing denominators, so they can be dropped. For each of the real roots of polynomial $\Delta_{n}$, we can determine the Cartesian position of the $v-k$ revolute pair centers of the ternary links, with respect to the $n$-ary link, using equations (9)-(13), equation (15), and the equation $\mathbf{p}_{1,3}=\mathbf{Z}_{1,4,3} \mathbf{p}_{1,4}$. This process leads up to $2^{k}$ combinations of locations for $P_{v-1}$ and $P_{v}$, and at least one of them must satisfy the distance imposed by the binary link connecting them.

\section{Examples}

\subsection{5-loop Watt-Baranov Truss}

Consider a 11-link Watt-Baranov truss. Since, in this case $k=5, v=15$, equation (17) reduces to 


$$
\operatorname{det}\left(-\mathbf{Q}_{11}+\mathbf{Z}_{1,2,15} \mathbf{Z}_{1,3,2}\right)=\frac{s_{14,15}}{s_{1,3}}
$$

where

$$
\begin{aligned}
\mathbf{Q}_{11} & =\mathbf{Z}_{1,10,13} \mathbf{Z}_{1,7,10} \mathbf{Z}_{1,4,7} \mathbf{Z}_{1,3,4}+\mathbf{Z}_{13,12,14} \mathbf{Z}_{13,11,12} \\
& \left(\mathbf{Z}_{1,7,10} \mathbf{Z}_{1,4,7} \mathbf{Z}_{1,3,4}+\mathbf{Z}_{10,9,11} \mathbf{Z}_{10,8,9}\left(\mathbf{Z}_{1,4,7} \mathbf{Z}_{1,3,4}\right.\right. \\
& \mathbf{Z}_{7,6,8} \mathbf{Z}_{7,5,6}\left(\mathbf{Z}_{1,3,4}+\mathbf{Z}_{4,3,5}+\left(\mathbf{I}-\mathbf{Z}_{1,3,4}\right)-\mathbf{Z}_{1,4,7} \mathbf{Z}_{1,3,4}\right) \\
& \left.\left.-\mathbf{Z}_{1,7,10} \mathbf{Z}_{1,4,7} \mathbf{Z}_{1,3,4}\right)-\mathbf{Z}_{1,10,13} \mathbf{Z}_{1,7,10} \mathbf{Z}_{1,4,7} \mathbf{Z}_{1,3,4}\right)
\end{aligned}
$$

and equations (22)-(25) reduce to

$$
\begin{aligned}
s_{5,7} & =\operatorname{det}\left(\mathbf{D}_{11_{1}}\right) s_{1,3} \\
s_{8,10} & =\operatorname{det}\left(\mathbf{D}_{11_{2}}\right) s_{1,3} \\
s_{11,13} & =\operatorname{det}\left(\mathbf{D}_{11_{3}}\right) s_{1,3} .
\end{aligned}
$$

where

$$
\begin{aligned}
\mathbf{D}_{11_{1}} & =-\mathbf{Z}_{1,3,4}-\mathbf{Z}_{4,3,5}\left(\mathbf{I}-\mathbf{Z}_{1,3,4}\right)+\mathbf{Z}_{1,4,7} \mathbf{Z}_{1,3,4} \\
\mathbf{D}_{11_{2}} & =-\mathbf{Z}_{1,4,7} \mathbf{Z}_{1,3,4}-\mathbf{Z}_{7,6,8} \mathbf{Z}_{7,5,6}\left(\mathbf{Z}_{1,3,4}+\mathbf{Z}_{4,3,5}(\mathbf{I}\right. \\
& \left.\left.-\mathbf{Z}_{1,3,4}\right)-\mathbf{Z}_{1,4,7} \mathbf{Z}_{1,3,4}\right)+\mathbf{Z}_{1,7,10} \mathbf{Z}_{1,4,7} \mathbf{Z}_{1,3,4} \\
\mathbf{D}_{11_{3}} & =-\mathbf{Z}_{1,7,10} \mathbf{Z}_{1,4,7} \mathbf{Z}_{1,3,4}-\mathbf{Z}_{10,9,11} \mathbf{Z}_{10,8,9}\left(\mathbf{Z}_{1,4,7} \mathbf{Z}_{1,3,4}\right. \\
& +\mathbf{Z}_{7,6,8} \mathbf{Z}_{7,5,6}\left(\mathbf{Z}_{1,3,4}+\mathbf{Z}_{4,3,5}\left(\mathbf{I}-\mathbf{Z}_{1,3,4}\right)-\mathbf{Z}_{1,4,7} \mathbf{Z}_{1,3,4}\right) \\
& \left.-\mathbf{Z}_{1,7,10} \mathbf{Z}_{1,4,7} \mathbf{Z}_{1,3,4}\right)+\mathbf{Z}_{1,10,13} \mathbf{Z}_{1,7,10} \mathbf{Z}_{1,4,7} \mathbf{Z}_{1,3,4}
\end{aligned}
$$

By expanding all the Cayley-Menger determinants involved in equations (28)-(30), we get

$$
\begin{aligned}
s_{5,7} & ={ }^{1} \Lambda_{1}+{ }^{1} \Lambda_{2} A_{1,3,4} \\
s_{8,10} & =\frac{1}{s_{5,7}}\left({ }^{2} \Lambda_{1}+{ }^{2} \Lambda_{2} A_{1,3,4}+{ }^{2} \Lambda_{3} A_{7,5,6}\right. \\
& \left.+{ }^{2} \Lambda_{4} A_{1,3,4} A_{7,5,6}\right) \\
s_{11,13} & =\frac{1}{s_{5,7} s_{8,10}}\left({ }^{3} \Lambda_{1}+{ }^{3} \Lambda_{2} A_{1,3,4}+{ }^{3} \Lambda_{3} A_{7,5,6}\right. \\
& +{ }^{3} \Lambda_{4} A_{10,8,9}+{ }^{3} \Lambda_{5} A_{1,3,4} A_{7,5,6} \\
& +{ }^{3} \Lambda_{6} A_{1,3,4} A_{10,8,9}+{ }^{3} \Lambda_{7} A_{7,5,6} A_{10,8,9} \\
& \left.+{ }^{3} \Lambda_{8} A_{1,3,4} A_{7,5,6} A_{10,8,9}\right)
\end{aligned}
$$

where

$$
\begin{aligned}
& A_{1,3,4}= \pm \frac{1}{2} \sqrt{\left[s_{1,3}-\left(d_{4,3}-d_{4,1}\right)^{2}\right]\left[\left(d_{4,3}+d_{4,1}\right)^{2}-s_{1,3}\right]} \\
& A_{7,5,6}= \pm \frac{1}{2} \sqrt{\left[s_{5,7}-\left(d_{6,5}-d_{6,7}\right)^{2}\right]\left[\left(d_{6,5}+d_{6,7}\right)^{2}-s_{5,7}\right]}
\end{aligned}
$$

$A_{10,8,9}=$

$$
\pm \frac{1}{2} \sqrt{\left[s_{8,10}-\left(d_{9,8}-d_{9,10}\right)^{2}\right]\left[\left(d_{9,8}+d_{9,10}\right)^{2}-s_{8,10}\right]}
$$

are the unknown areas of the triangles $P_{1} P_{3} P_{4}, P_{7} P_{5} P_{6}$, and $P_{10} P_{8} P_{9}$, respectively, ${ }^{1} \Lambda_{1},{ }^{1} \Lambda_{2}$ are polynomials in $s_{1,3},{ }^{2} \Lambda_{i}$, $i=1, \ldots, 4$ are polynomials in $s_{1,3}$ and $s_{5,7}$, and ${ }^{3} \Lambda_{i}, i=$ $1, \ldots, 8$ are polynomials in $s_{1,3}, s_{5,7}$, and $s_{8,10}$.

Similarly, by expanding all the Cayley-Menger determinants in equation (27), we get

$$
\frac{1}{s_{1,3}^{2} s_{5,7} s_{8,10} s_{11,13}} \Psi=\frac{s_{14,15}}{s_{1,3}}
$$

that is,

$$
\Psi=s_{1,3} s_{5,7} s_{8,10} s_{11,13} s_{14,15},
$$

where

$$
\begin{aligned}
\Psi & =\Psi_{1}+\Psi_{2} A_{1,2,3}+\Psi_{3} A_{1,3,4}+\Psi_{4} A_{7,5,6}+\Psi_{5} A_{10,8,9} \\
& +\Psi_{6} A_{13,11,12}+\Psi_{7} A_{1,2,3} A_{1,3,4}+\Psi_{8} A_{1,2,3} A_{7,5,6} \\
& +\Psi_{9} A_{1,2,3} A_{10,8,9}+\Psi_{10} A_{1,2,3} A_{13,11,12}+\Psi_{11} A_{1,3,4} A_{7,5,6} \\
& +\Psi_{12} A_{1,3,4} A_{10,8,9}+\ldots+\Psi_{31} A_{1,3,4} A_{7,5,6} A_{10,8,9} A_{13,11,12} \\
& +\Psi_{32} A_{1,2,3} A_{1,3,4} A_{7,5,6} A_{10,8,9} A_{13,11,12},
\end{aligned}
$$

with $\Psi_{i}, i=1, \ldots, 2^{5}$, polynomials in $s_{1,3}, s_{5,7}, s_{8,10}$, and $s_{11,13}$.

Now, by expressing equation (35) as a linear equation in $A_{13,11,12}$-i.e., $a+b A_{13,11,12}=0$, properly squaring it i.e., $a^{2}-b^{2} A_{13,11,12}^{2}=0$, and replacing equation (33) in the result, a radical equation in $s_{1,3}, s_{5,7}$, and $s_{8,10}$ is obtained. Repeating this process for $A_{10,8,9}$ and then for $A_{7,5,6}$, we get the scalar radical equation

$$
\Phi_{1}+\Phi_{2} A_{1,2,3}+\Phi_{3} A_{1,3,4}+\Phi_{4} A_{1,2,3} A_{1,3,4}=0
$$

where $\Phi_{1}, \Phi_{2}, \Phi_{3}$ and $\Phi_{4}$ are polynomials in a single variable: $s_{1,3}$. If the last procedure is applied to equations (31), (32), and (33), we get polynomials in $s_{1,3}$ and $s_{5,7}$, say $P_{1}\left(s_{1,3}, s_{5,7}\right), s_{1,3}$ and $s_{8,10}$, say $P_{2}\left(s_{1,3}, s_{8,10}\right)$, and $s_{1,3}$ and $s_{11,13}$, say $P_{3}\left(s_{1,3}, s_{11,13}\right)$, respectively.

Finally, the square roots in (36) can be eliminated by properly twice squaring it. This operation yields

$$
\begin{aligned}
& -\Phi_{4}^{4} A_{1,2,3}^{4} A_{1,3,4}^{4}+2 \Phi_{4}^{2} \Phi_{2}^{2} A_{1,2,3}^{4} A_{1,3,4}^{2}+2 \Phi_{4}^{2} \Phi_{3}^{2} A_{1,2,3}^{2} A_{1,3,4}^{4} \\
& -\Phi_{2}^{4} A_{1,2,3}^{4}-\Phi_{3}^{4} A_{1,3,4}^{4}-\Phi_{1}^{4}+\left(2 \Phi_{2}^{2} \Phi_{3}^{2}-8 \Phi_{2} \Phi_{3} \Phi_{4} \Phi_{1}\right. \\
& \left.+2 \Phi_{4}^{2} \Phi_{1}^{2}\right) A_{1,2,3}^{2} A_{1,3,4}^{2}+2 \Phi_{1}^{2} \Phi_{2}^{2} A_{1,2,3}^{2}+2 \Phi_{1}^{2} \Phi_{3}^{2} A_{1,3,4}^{2}=0
\end{aligned}
$$

which, when fully expanded, leads to

$$
\begin{array}{r}
s_{1,3}^{16} P_{1}\left(s_{1,3}, 0\right)^{8} P_{2}\left(s_{1,3}, 0\right)^{4} P_{3}\left(s_{1,3}, 0\right)^{2} \Delta_{11}=0 \\
s_{1,3}^{16} s_{5,7}^{8} s_{8,10}^{4} s_{11,13}^{2} \Delta_{11}=0
\end{array}
$$


where $\Delta_{11}$ is a polynomial in $s_{1,3}$ of degree 62 . The extraneous roots at $s_{5,7}=0, s_{8,10}=0$, and $s_{11,13}=0$ were introduced when clearing denominators to obtain equation (35), so they can be dropped.

Finally, let us suppose that $s_{1,2}=40, s_{1,4}=13, s_{1,7}=26$, $s_{1,10}=34, s_{1,13}=17, s_{1,15}=13, s_{2,3}=50, s_{2,15}=17$, $s_{3,4}=81, s_{3,5}=9, s_{4,5}=90, s_{4,7}=13, s_{4,10}=49, s_{4,13}=$ $52, s_{5,6}=125, s_{6,7}=40, s_{6,8}=9, s_{7,8}=37, s_{7,10}=20$, $s_{7,13}=45, s_{8,9}=136, s_{9,10}=53, s_{9,11}=9, s_{10,11}=50$, $s_{10,13}=17, s_{11,12}=181, s_{12,13}=50, s_{12,14}=9, s_{13,14}=65$, and $s_{14,15}=29$. Then, proceeding as explained above, we obtain the characteristic polynomial

$$
\begin{aligned}
& s_{1,3}{ }^{62}-4091.5078 s_{1,3}{ }^{61}+8.307410^{6} s_{1,3}{ }^{60}-1.118610^{10} s_{1,3}{ }^{59}+1.126010^{13} s_{1,3}{ }^{58} \\
& -9.051910^{15} s_{1,3}{ }^{57}+6.060410^{18} s_{1,3}{ }^{56}-3.477610^{21} s_{1,3}{ }^{55}+1.746110^{24} s_{1,3}{ }^{54} \\
& -7.789410^{26} s_{1,3}{ }^{53}+3.123810^{29} s_{1,3}{ }^{52}-1.136310^{32} s_{1,3}{ }^{51}+3.775110^{34} s_{1,3}{ }^{50} \\
& -1.151310^{37} s_{1,3}{ }^{49}+3.236010^{39} s_{1,3}{ }^{48}-8.404410^{41} s_{1,3}{ }^{47}+2.020810^{44} s_{1,3}{ }^{46} \\
& -4.504010^{46} s_{1,3}{ }^{45}+9.312910^{48} s_{1,3}{ }^{44}-1.787410^{51} s_{1,3}{ }^{43}+3.185510^{53} s_{1,3}{ }^{42} \\
& -5.273010^{55} s_{1,3}{ }^{41}+8.109210^{57} s_{1,3}{ }^{40}-1.158910^{60} s_{1,3}{ }^{39}+1.539110^{62} s_{1,3}{ }^{38} \\
& -1.900210^{64} s_{1,3}{ }^{37}+2.180710^{66} s_{1,3}{ }^{36}-2.326510^{68} s_{1,3}{ }^{35}+2.307310^{70} s_{1,3}{ }^{34} \\
& -2.126710^{72} s_{1,3}{ }^{33}+1.821510^{74} s_{1,3}{ }^{32}-1.449210^{76} s_{1,3}{ }^{31}+1.070410^{78} s_{1,3}{ }^{30} \\
& -7.336610^{79} s_{1,3}{ }^{29}+4.662310^{81} s_{1,3}{ }^{28}-2.744710^{83} s_{1,3}{ }^{27}+1.495210^{85} s_{1,3}{ }^{26} \\
& -7.529110^{86} s_{1,3}{ }^{25}+3.499210^{88} s_{1,3}{ }^{24}-1.498710^{90} s_{1,3}{ }^{23}+5.904110^{91} s_{1,3}{ }^{22} \\
& -2.135310^{93} s_{1,3}{ }^{21}+7.073110^{94} s_{1,3}{ }^{20}-2.140710^{96} s_{1,3}{ }^{19}+5.903210^{97} s_{1,3}{ }^{18} \\
& -1.479110^{99} s_{1,3}{ }^{17}+3.35710^{100} s_{1,3}{ }^{16}-6.881910^{101} s_{1,3}{ }^{15}+1.27110^{103} s_{1,3}{ }^{14} \\
& -2.11110^{104} s_{1,3}{ }^{13}+3.14910^{105} s_{1,3}{ }^{12}-4.222610^{106} s_{1,3}{ }^{11}+5.099710^{107} s_{1,3}{ }^{10} \\
& -5.552610^{108} s_{1,3}{ }^{9}+5.432810^{109} s_{1,3}{ }^{8}-4.716610^{110} s_{1,3}{ }^{7}+3.539810^{111} s_{1,3}{ }^{6} \\
& -2.202910^{112} s_{1,3}{ }^{5}+1.072110^{113} s_{1,3}{ }^{4}-3.758610^{113} s_{1,3}{ }^{3}+8.417710^{113} s_{1,3}{ }^{2} \\
& -1.025810^{114} s_{1,3}+7.386210^{113}=0 .
\end{aligned}
$$

This polynomial has 16 real roots. The values of these roots as well as the corresponding assembly modes, for the case in which $P_{1}=(12,10)^{T}, P_{4}=(10,13)^{T}, P_{7}=(13,15)^{T}$, $P_{10}=(17,13)^{T}$, and $P_{13}=(16,9)^{T}$, appear in Fig. 4 .

The coefficients of the above polynomial have to be computed in rational arithmetic. Otherwise, numerical problems make impracticable the correct computation of its roots. Although these coefficients are given here in floating point arithmetic for space limitation reasons, they could be of interest for comparison with other possible methods.

\subsection{6-loop Watt-Baranov Truss}

Let us consider a 13-link Watt-Baranov truss where $s_{1,2}=58, s_{1,4}=18, s_{1,7}=40, s_{1,10}=53, s_{1,13}=50, s_{1,16}=$ $20, s_{1,18}=41, s_{2,3}=52, s_{2,18}=13, s_{3,4}=64, s_{3,5}=18$, $s_{4,5}=34, s_{4,7}=10, s_{4,10}=41, s_{4,13}=68, s_{4,16}=50, s_{5,6}=$ $50, s_{6,7}=74, s_{6,8}=10, s_{7,8}=68, s_{7,10}=17, s_{7,13}=50$, $s_{7,16}=52, s_{8,9}=65, s_{9,10}=68, s_{9,11}=9, s_{10,11}=89$, $s_{10,13}=13, s_{10,16}=29, s_{11,12}=61, s_{12,13}=65, s_{12,14}=26$, $s_{13,14}=65, s_{13,16}=10, s_{14,15}=113, s_{15,16}=40, s_{15,17}=13$, $s_{16,17}=81$, and $s_{17,18}=68$. Then, proceeding as explained in the previous example, the following characteristic polynomial is obtained

$$
\begin{aligned}
& s_{1,3}{ }^{126}-9.433610^{3} s_{1,3}{ }^{125}+4.396510^{7} s_{1,3}{ }^{124}-1.349910^{11} s_{1,3}{ }^{123}+3.072710^{14} s_{1,3}{ }^{122} \\
& -5.532610^{17} s_{1,3}{ }^{121}+8.211210^{20} s_{1,3}{ }^{120}-1.033510^{24} s_{1,3}{ }^{119}+1.126510^{27} s_{1,3}{ }^{118} \\
& -1.080410^{30} s_{1,3}{ }^{117}+9.233910^{32} s_{1,3}{ }^{116}-7.105310^{35} s_{1,3}{ }^{115}+4.964510^{38} s_{1,3}{ }^{114} \\
& -3.172710^{41} s_{1,3}{ }^{113}+1.866310^{44} s_{1,3}{ }^{112}-1.016210^{47} s_{1,3}{ }^{111}+5.148210^{49} s_{1,3}{ }^{110} \\
& -2.438210^{52} s_{1,3}{ }^{109}+1.084310^{55} s_{1,3}{ }^{108}-4.547410^{57} s_{1,3}{ }^{107}+1.805510^{60} s_{1,3}{ }^{106} \\
& -6.812410^{62} s_{1,3}{ }^{105}+2.450810^{65} s_{1,3}{ }^{104}-8.431910^{67} s_{1,3}{ }^{103}+2.781310^{70} s_{1,3}{ }^{102}
\end{aligned}
$$




$$
\begin{aligned}
& -8.813910^{72} s_{1,3}{ }^{101}+2.687410^{75} s_{1,3}{ }^{100}-7.892310^{77} s_{1,3}{ }^{99}+2.233710^{80} s_{1,3}{ }^{98} \\
& -6.094210^{82} s_{1,3}{ }^{97}+1.602610^{85} s_{1,3}{ }^{96}-4.060610^{87} s_{1,3}{ }^{95}+9.909010^{89} s_{1,3}{ }^{94} \\
& -2.327410^{92} s_{1,3}{ }^{93}+5.257910^{94} s_{1,3}{ }^{92}-1.141810^{97} s_{1,3}{ }^{91}+2.381610^{99} s_{1,3}{ }^{90} \\
& -4.768810^{101} s_{1,3}{ }^{89}+9.161310^{103} s_{1,3}{ }^{88}-1.687710^{106} s_{1,3}{ }^{87}+2.980410^{108} s_{1,3}{ }^{86} \\
& -5.043410^{110} s_{1,3}{ }^{85}+8.176010^{112} s_{1,3}{ }^{84}-1.269510^{115} s_{1,3}{ }^{83}+1.887910^{117} s_{1,3}{ }^{82} \\
& -2.688610^{119} s_{1,3}{ }^{81}+3.666510^{121} s_{1,3}{ }^{80}-4.788410^{123} s_{1,3}{ }^{79}+5.988710^{125} s_{1,3}{ }^{78} \\
& -7.173310^{127} s_{1,3}{ }^{77}+8.229610^{129} s_{1,3}{ }^{76}-9.043510^{131} s_{1,3}{ }^{75}+9.519910^{133} s_{1,3}{ }^{74} \\
& -9.600510^{135} s_{1,3}{ }^{73}+9.275810^{137} s_{1,3}{ }^{72}-8.586810^{139} s_{1,3}{ }^{71}+7.616310^{141} s_{1,3}{ }^{70} \\
& -6.472910^{143} s_{1,3}{ }^{69}+5.271110^{145} s_{1,3}{ }^{68}-4.112810^{147} s_{1,3}{ }^{67}+3.074610^{149} s_{1,3}{ }^{66} \\
& -2.202010^{151} s_{1,3}{ }^{65}+1.510710^{153} s_{1,3}{ }^{64}-9.926610^{154} s_{1,3}{ }^{63}+6.246210^{156} s_{1,3}{ }^{62} \\
& -3.762810^{158} s_{1,3}{ }^{61}+2.169610^{160} s_{1,3}{ }^{60}-1.196910^{162} s_{1,3}{ }^{59}+6.315410^{163} s_{1,3}{ }^{58} \\
& -3.185610^{165} s_{1,3}{ }^{57}+1.535310^{167} s_{1,3}{ }^{56}-7.065010^{168} s_{1,3}{ }^{55}+3.102010^{170} s_{1,3}{ }^{54} \\
& -1.298410^{172} s_{1,3}{ }^{53}+5.174810^{173} s_{1,3}{ }^{52}-1.961510^{175} s_{1,3}{ }^{51}+7.059510^{176} s_{1,3}{ }^{50} \\
& -2.407910^{178} s_{1,3}{ }^{49}+7.764110^{179} s_{1,3}{ }^{48}-2.359110^{181} s_{1,3}{ }^{47}+6.726110^{182} s_{1,3}{ }^{46} \\
& -1.788610^{184} s_{1,3}{ }^{45}+4.396110^{185} s_{1,3}{ }^{44}-9.844210^{186} s_{1,3}{ }^{43}+1.956110^{188} s_{1,3}{ }^{42} \\
& -3.255610^{189} s_{1,3}{ }^{41}+3.774610^{190} s_{1,3}{ }^{40}+3.778910^{190} s_{1,3}{ }^{39}-1.903810^{193} s_{1,3}{ }^{38} \\
& +7.173410^{194} s_{1,3}{ }^{37}-1.875110^{196} s_{1,3}{ }^{36}+3.883410^{197} s_{1,3}{ }^{35}-6.309910^{198} s_{1,3}{ }^{34} \\
& +6.690610^{199} s_{1,3}{ }^{33}+2.038310^{200} s_{1,3}{ }^{32}-3.535110^{202} s_{1,3}{ }^{31}+1.213510^{204} s_{1,3}{ }^{30} \\
& -3.031610^{205} s_{1,3}{ }^{29}+6.359510^{206} s_{1,3}{ }^{28}-1.174910^{208} s_{1,3}{ }^{27}+1.953510^{209} s_{1,3}{ }^{26} \\
& -2.956010^{210} s_{1,3}{ }^{25}+4.096210^{211} s_{1,3}{ }^{24}-5.216210^{212} s_{1,3}{ }^{23}+6.114610^{213} s_{1,3} 22 \\
& -6.602310^{214} s_{1,3}{ }^{21}+6.565310^{215} s_{1,3}{ }^{20}-6.007310^{216} s_{1,3}{ }^{19}+5.051410^{217} s_{1,3}{ }^{18} \\
& -3.897010^{218} s_{1,3}{ }^{17}+2.752810^{219} s_{1,3}{ }^{16}-1.776510^{220} s_{1,3}{ }^{15}+1.045010^{221} s_{1,3}{ }^{14} \\
& -5.588610^{221} s_{1,3}{ }^{13}+2.710610^{222} s_{1,3}{ }^{12}-1.189310^{223} s_{1,3}{ }^{11}+4.707910^{223} s_{1,3}{ }^{10} \\
& -1.675710^{224} s_{1,3}{ }^{9}+5.340210^{224} s_{1,3}{ }^{8}-1.513910^{225} s_{1,3}{ }^{7}+3.781110^{225} s_{1,3}{ }^{6} \\
& -8.203010^{225} s_{1,3}{ }^{5}+1.513810^{226} s_{1,3}{ }^{4}-2.301010^{226} s_{1,3}{ }^{3}+2.726510^{226} s_{1,3}{ }^{2} \\
& -2.255610^{226} s_{1,3}+9.789310^{225}=0 \text {. }
\end{aligned}
$$

This polynomial, that was computed using exact rational arithmetic and is presented here only for comparison purposes of eventual future works, has 76 real roots. The values of these roots as well as the corresponding configurations, for the case in which $P_{1}=(12,8)^{T}, P_{4}=(9,11)^{T}$, $P_{7}=(10,14)^{T}, P_{10}=(14,15)^{T}, P_{13}=(17,13)^{T}$, and $P_{16}=$ $(16,10)^{T}$, appear in Figs. 5, 6, and 7.

\section{Conclusion}

Given a Watt-Baranov truss, it has been shown how a scalar radical equation - which is satisfied if, and only if, it is assemblable - can be straightforwardly derived using bilaterations, independently of the number of its kinematic loops. Clearing radicals from this equation leads to the characteristic polynomial of the corresponding Watt-Baranov truss. Although conceptually simple, this clearing operation is computationally costly as it yields an exponential number of terms with the number of involved bilaterations. The whole process has been carried out for Watt-Baranov trusses with up to six loops and two examples have been presented. Obtaining the characteristic polynomial of a Watt-Baranov truss with more than six loops becomes a huge task. This suggest the convenience of working with the compact expression including radicals whenever possible, depending on the application.

\section{Achnowledgements}

We gratefully acknowledge the financial support of the Autonomous Government of Catalonia through the VALTEC program, cofinanced with FEDER funds, and the Colombian Ministry of Communications and Colfuturo through the ICT National Plan of Colombia. 

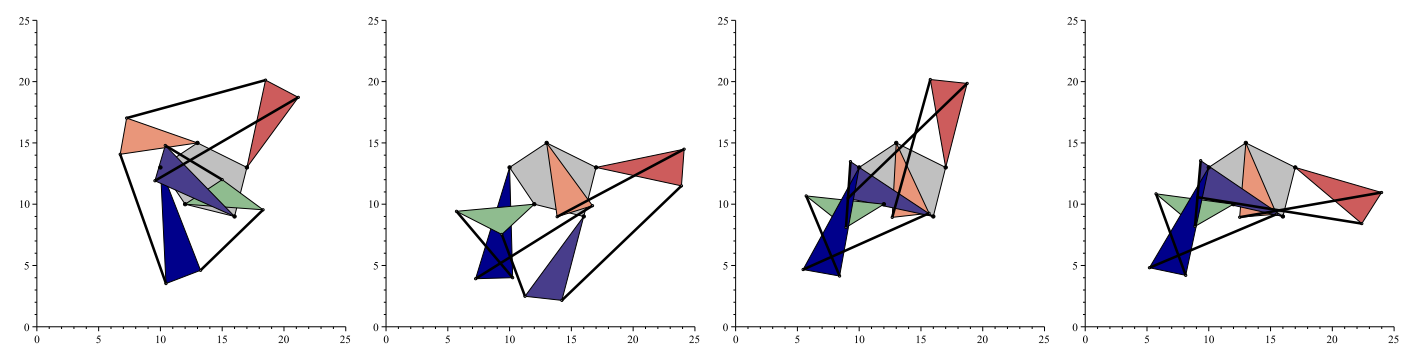

$s_{1,3}=30.6486$

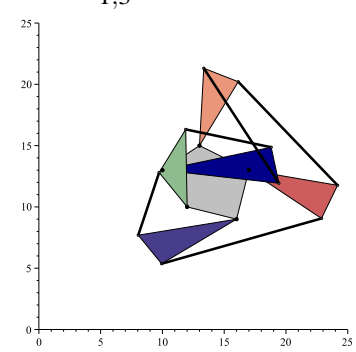

$s_{1,3}=39.0249$

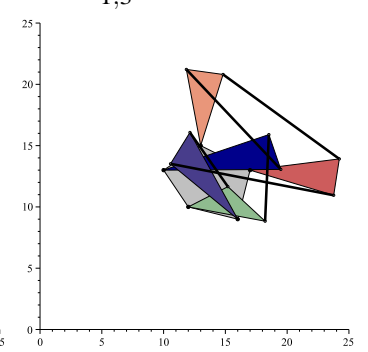

$s_{1,3}=47.1860$

$s_{1,3}=48.6406$

$s_{1,3}=69.9863$

$s_{1,3}=77.3161$
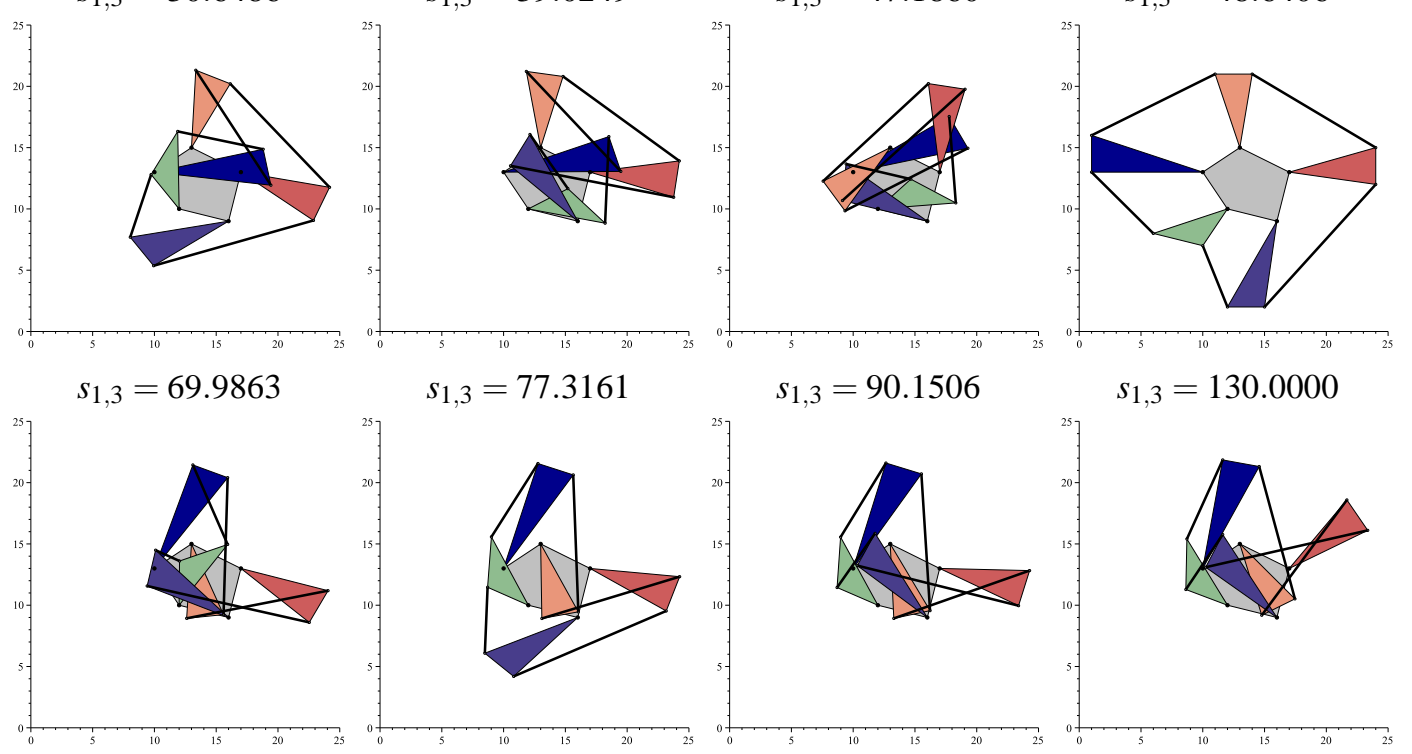

$s_{1,3}=90.1506$

$s_{1,3}=130.0000$
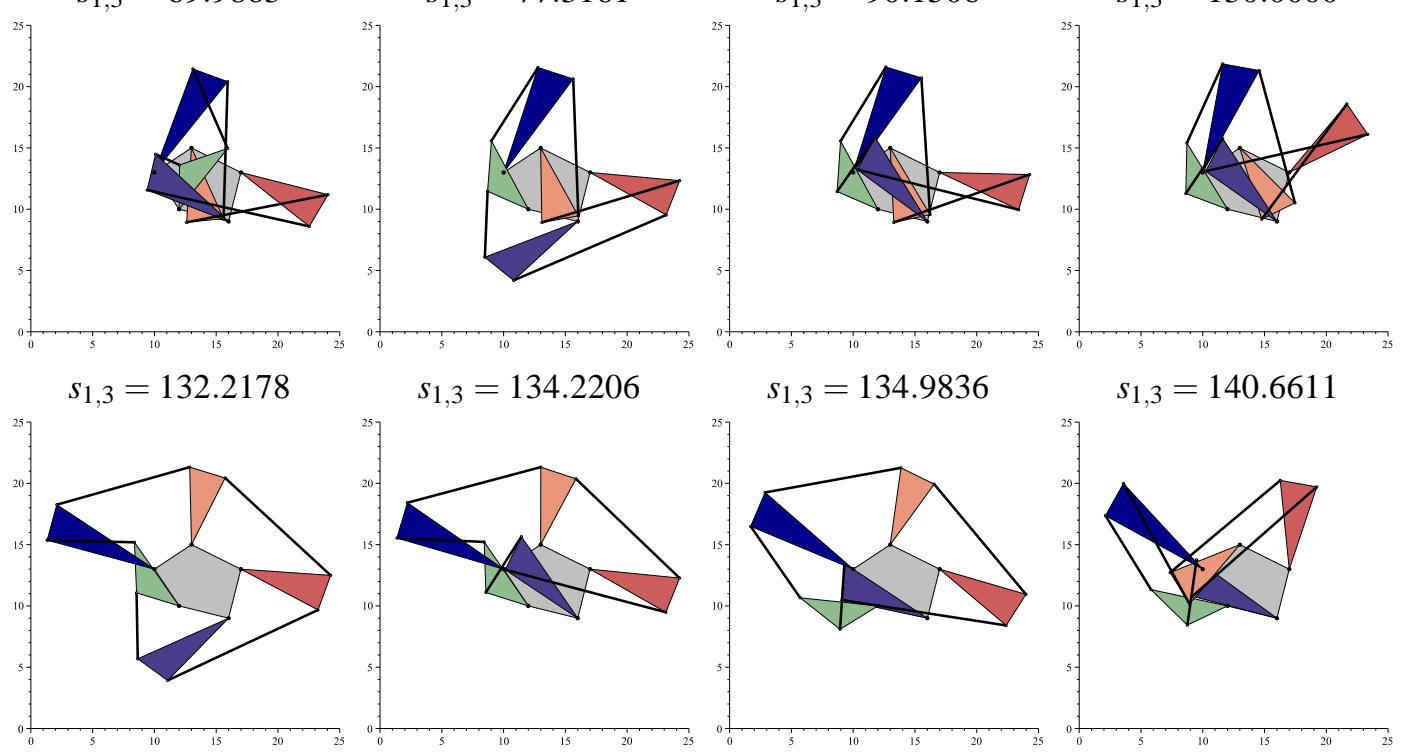

$s_{1,3}=134.9836$

$s_{1,3}=140.6611$

$s_{1,3}=142.9286$

$s_{1,3}=143.7773$

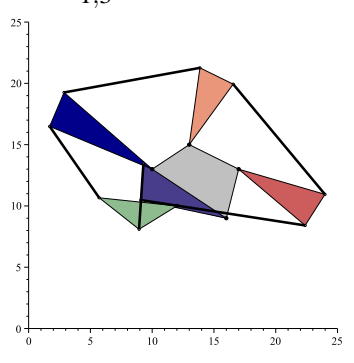

$s_{1,3}=148.1286$

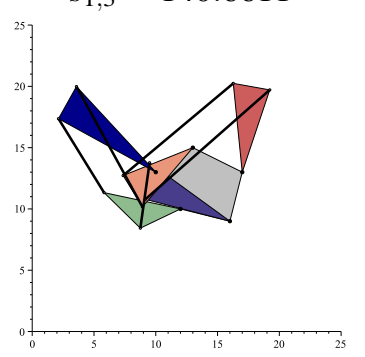

$s_{1,3}=151.6614$

Fig. 4. The assembly modes of the analyzed 11-link Watt-Baranov truss

\section{References}

[1] Galletti, C., 1986. "A note on modular approaches to planar linkage kinematic analysis". Mechanism and Machine Theory, 21(5), pp. 385 - 391.

[2] Ceresole, E., Fanghella, P., and Galletti, C., 1996. "Assur's groups, AKCs, basic trusses, SOCs, etc.: Modular kinematics of planar linkages, 96-DETC/MECH1027'. In Proceedings of the ASME 1996 International Design Engineering Technical Conferences and Computers in Engineering Conference.

[3] Peisach, E., 2008. "On Assur groups, Baranov trusses, Grübler chains, planar linkages and on their structural (number) synthesis". In The 22th Working Meeting of the IFToMM Permanent Commission for Standardization of Terminology, pp. $33-41$.

[4] Manolescu, N., 1973. "A method based on Baranov trusses, and using graph theory to find the set of planar jointed kinematic chains and mechanisms". Mechanism and Machine Theory, $8(1)$, pp. 3 - 22.

[5] Baranov, G., 1952. "Classification, formation, kinematics, and kinetostatics of mechanisms with pairs of the first kind (in Russian)". In Proceedings of Seminar on the Theory of Machines and Mechanisms, Moscow, Vol. 2, pp. $15-39$.

[6] Yang, T., and Yao, F., 1994. "Topological characteristics and automatic generation of structural synthesis of planar mechanisms based on the ordered singleopened-chains". In ASME Mechanism Synthesis and Analysis, DE-Vol. 70, pp. 67 - 74.

[7] Rojas, N., and Thomas, F., 2011. "Distance-based position analysis of the three seven-link Assur kinematic chains". Mechanism and Machine Theory, 46(2), 

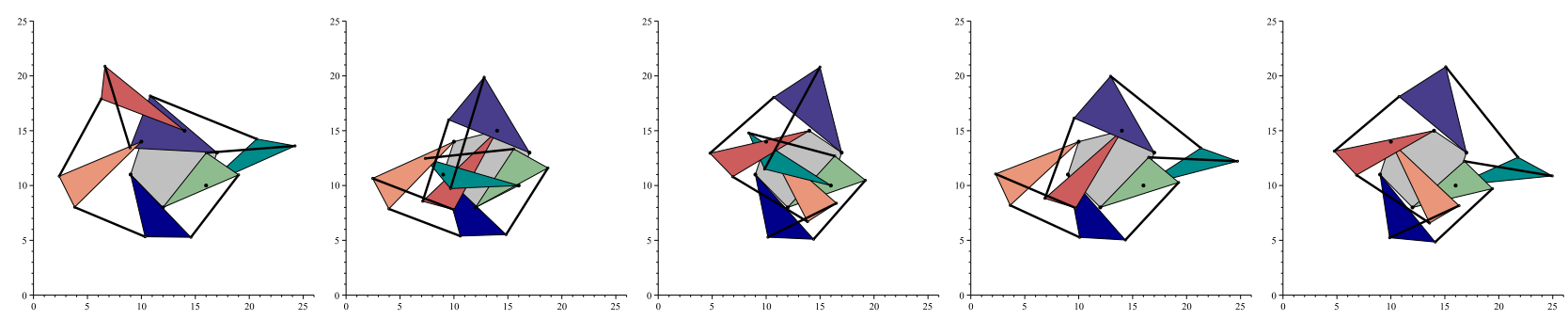

$s_{1,3}=14.1226$
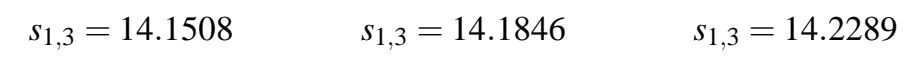

$s_{1,3}=14.4123$
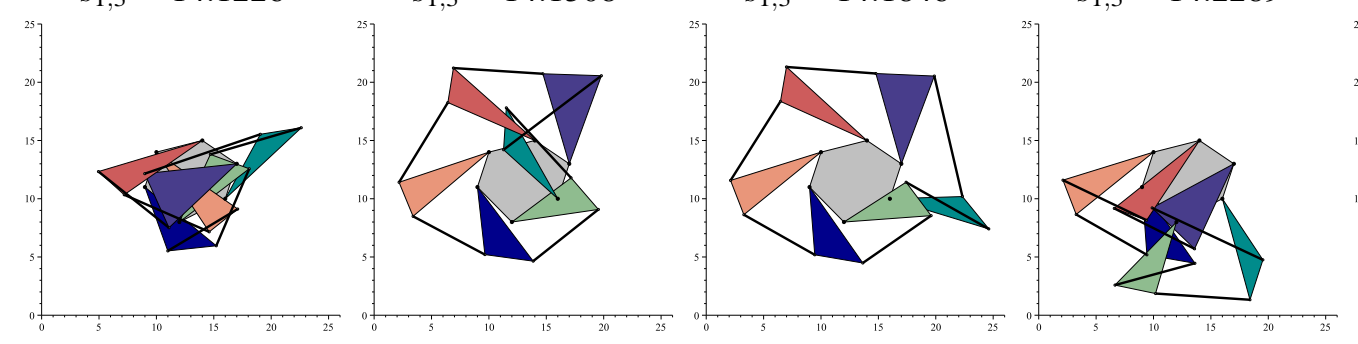

$s_{1,3}=14.4852$

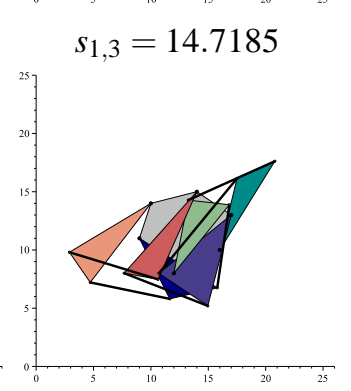

$s_{1,3}=15.0578$

$s_{1,3}=15.1158$
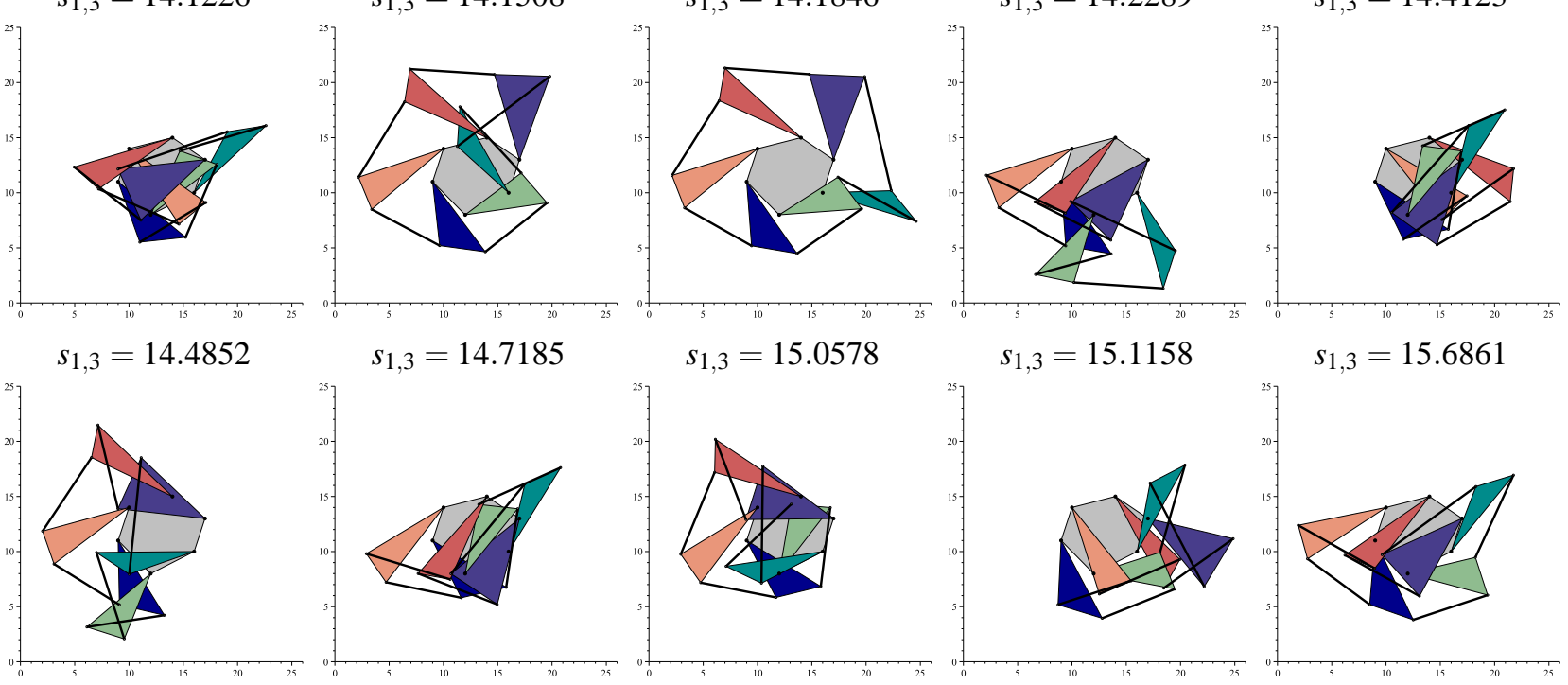

$s_{1,3}=15.6861$

$s_{1,3}=15.8268$

$s_{1,3}=15.8328$

$s_{1,3}=16.0193$

$s_{1,3}=17.0205$
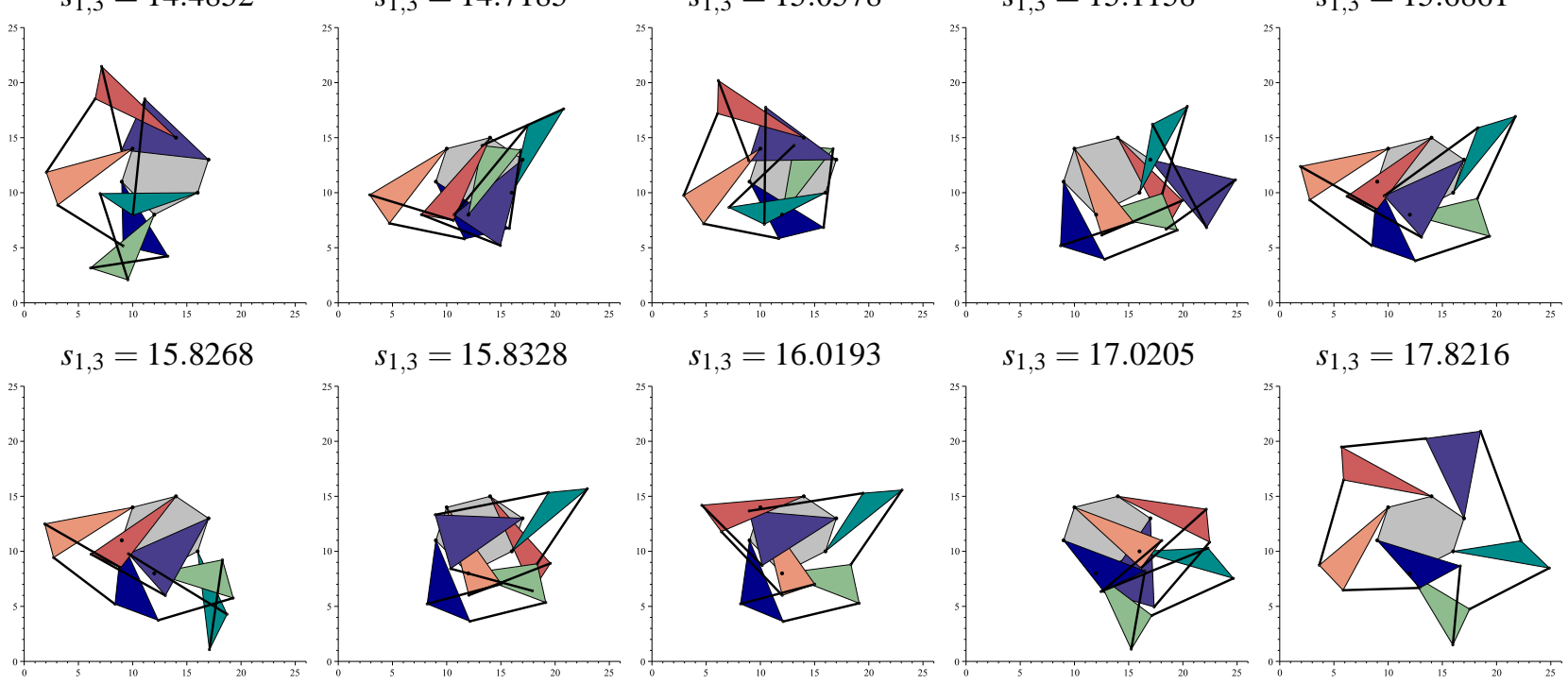

$s_{1,3}=17.8216$

$s_{1,3}=18.2916$
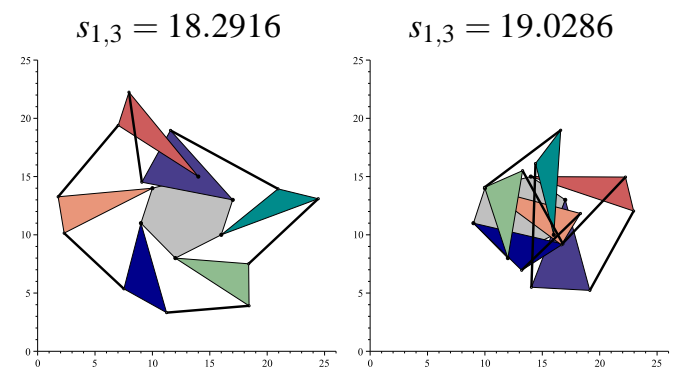

$s_{1,3}=19.1581$

$s_{1,3}=22.5020$

$s_{1,3}=24.4759$

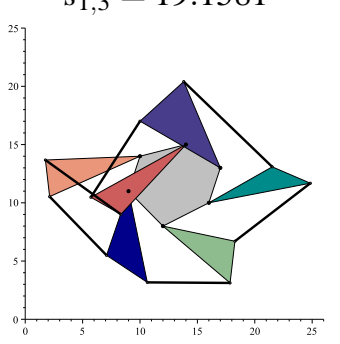

$s_{1,3}=20.2651$

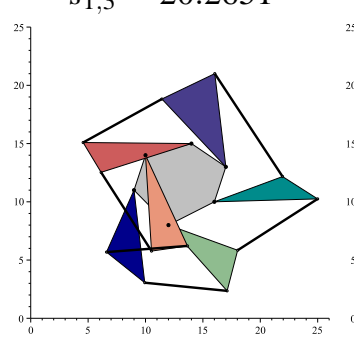

$s_{1,3}=25.1965$

$s_{1,3}=28.7237$

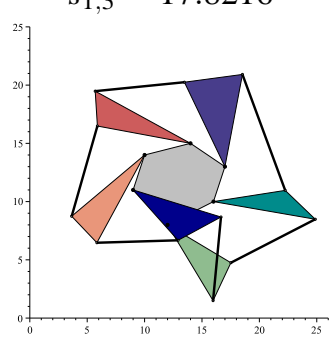

$s_{1,3}=22.1260$

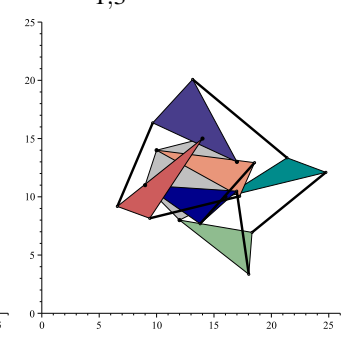

$s_{1,3}=31.0109$
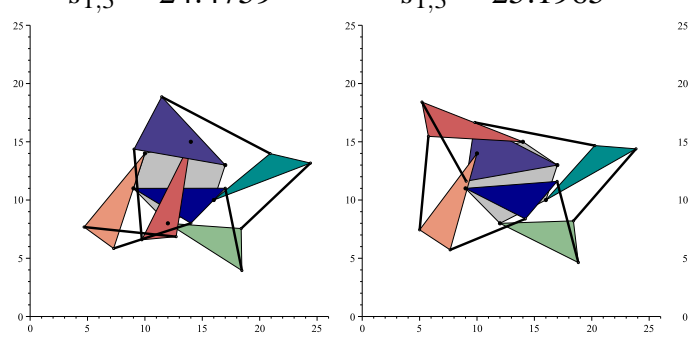

$s_{1,3}=34.0693$

$s_{1,3}=37.7304$
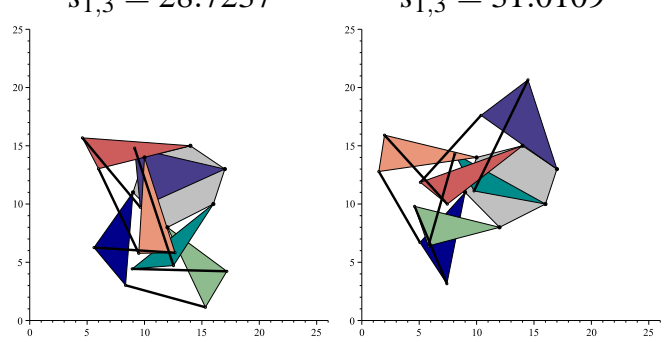

$s_{1,3}=38.0758$

$s_{1,3}=44.4875$

Fig. 5. The assembly modes of the analyzed 13-link Watt-Baranov truss (Part 1) 

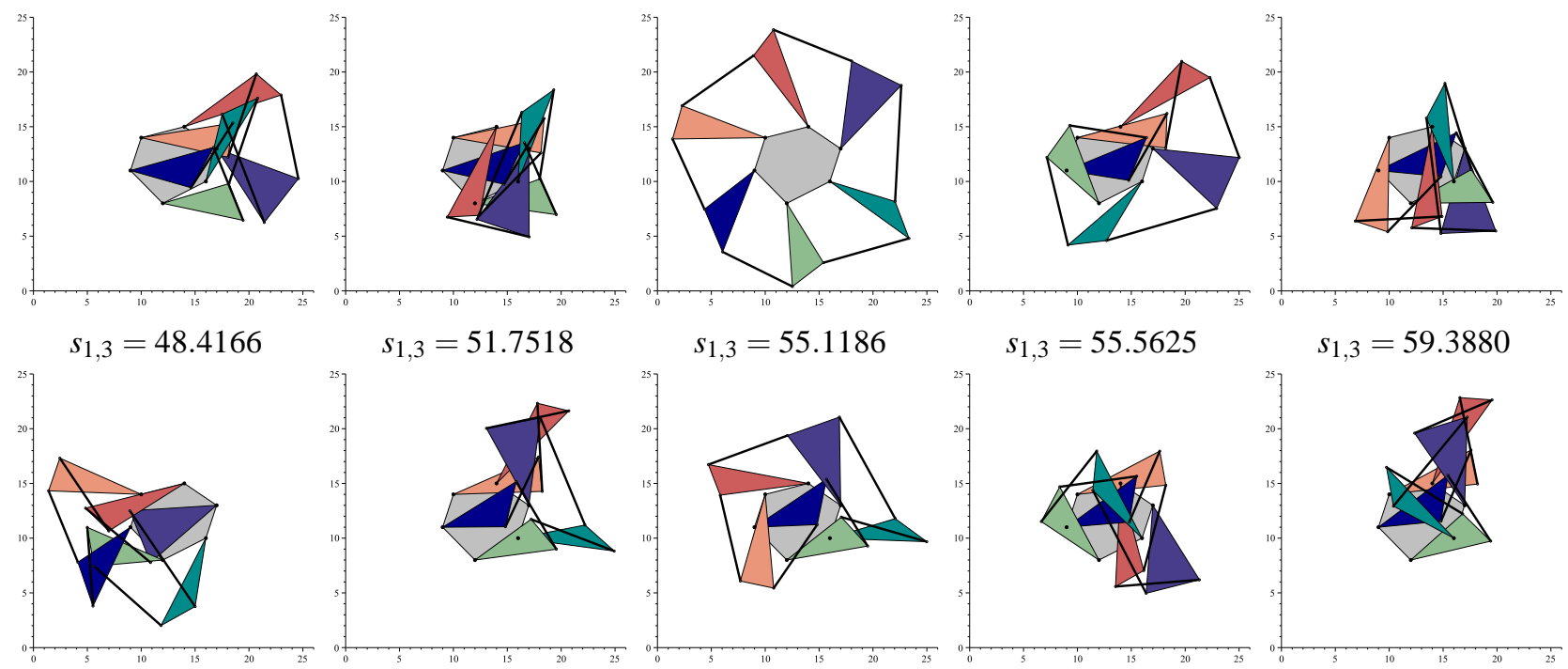

$s_{1,3}=51.7518$

$s_{1,3}=55.1186$

$s_{1,3}=55.5625$

$s_{1,3}=59.3880$

$s_{1,3}=59.6226$
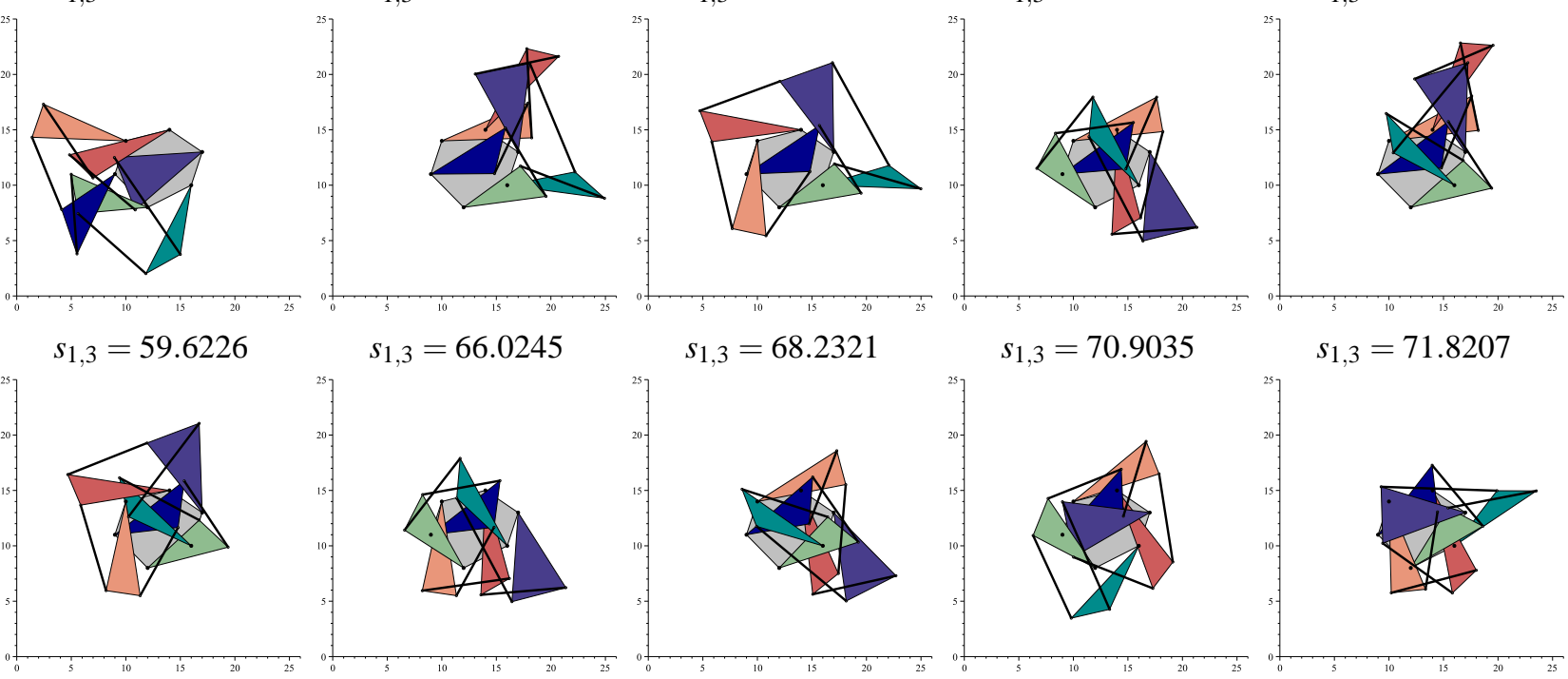

$s_{1,3}=66.0245$

$s_{1,3}=68.2321$

$s_{1,3}=70.9035$

$s_{1,3}=71.8207$
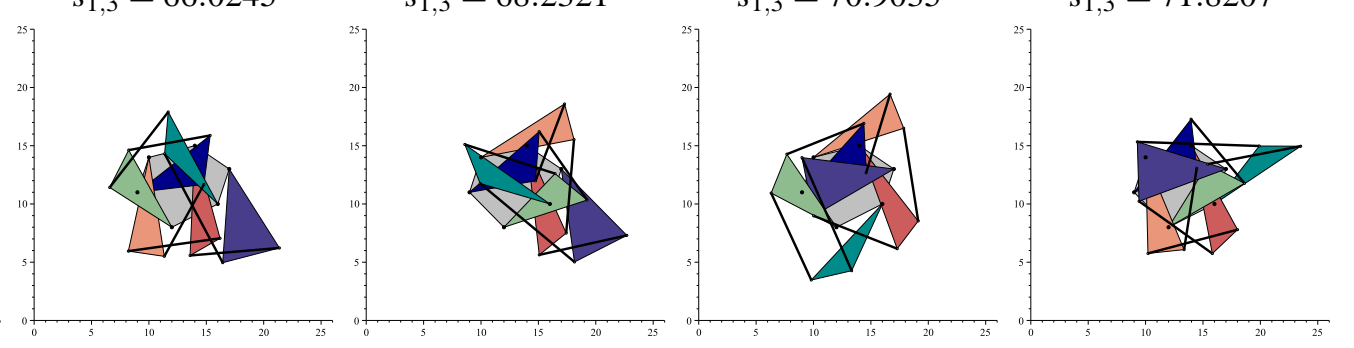

$s_{1,3}=73.0411$

$s_{1,3}=73.2584$

$s_{1,3}=76.8581$

$s_{1,3}=85.1396$

$s_{1,3}=89.9046$
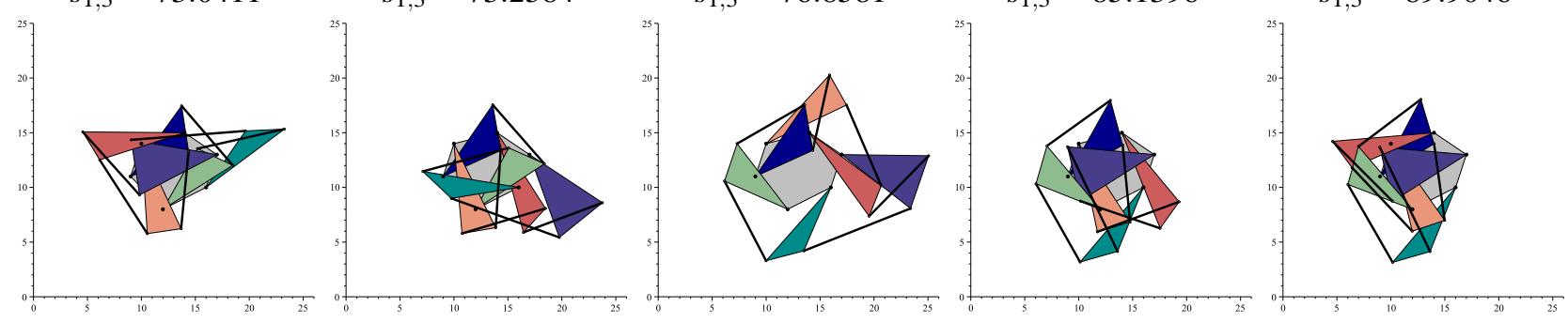

$s_{1,3}=92.3656$
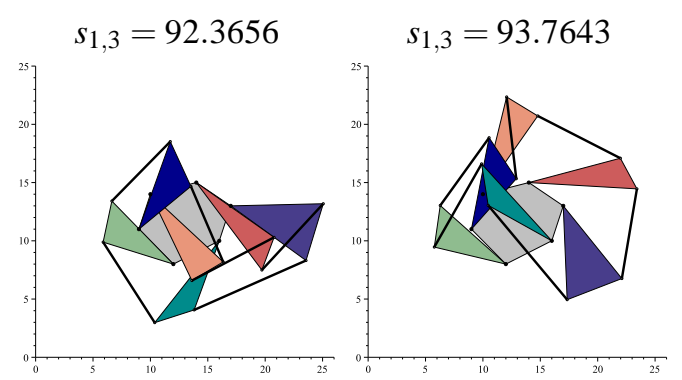

$s_{1,3}=93.9066$

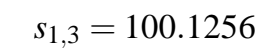

$s_{1,3}=101.6541$

$s_{1,3}=110.7250$

$s_{1,3}=119.8610$
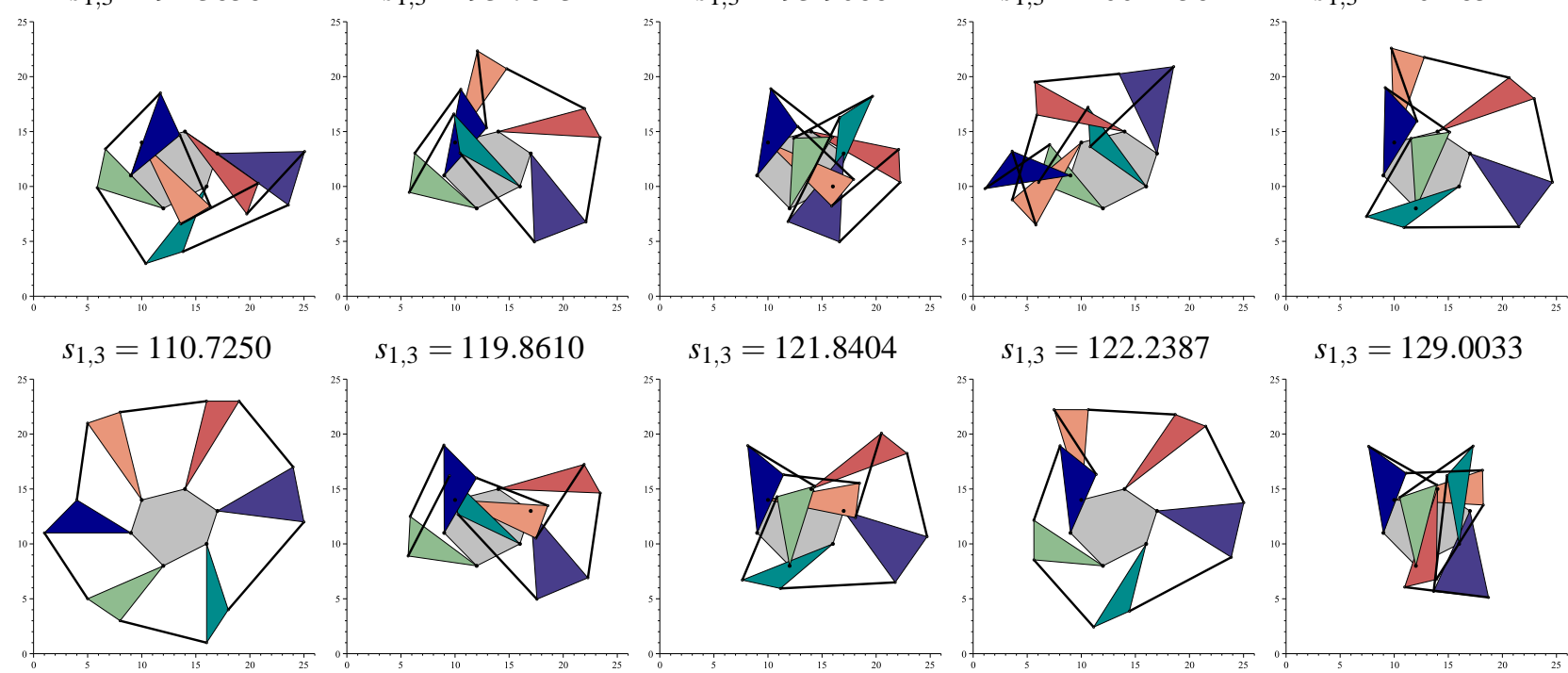

$s_{1,3}=130.0000$

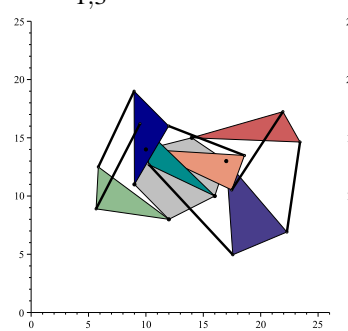

$s_{1,3}=121.8404$

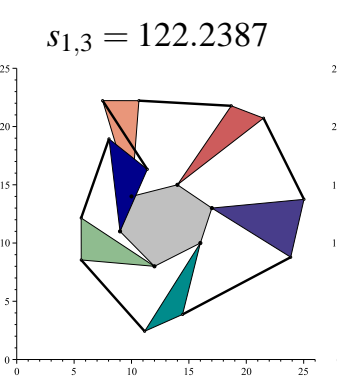

$s_{1,3}=130.1666$

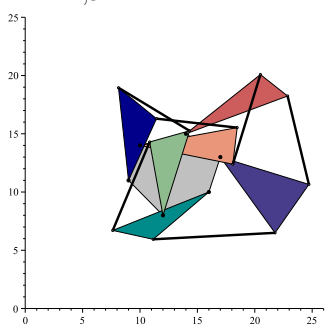

$s_{1,3}=134.9545$

$s_{1,3}=135.4180$

$s_{1,3}=137.5075$

Fig. 6. The assembly modes of the analyzed 13-link Watt-Baranov truss (Part 2) 

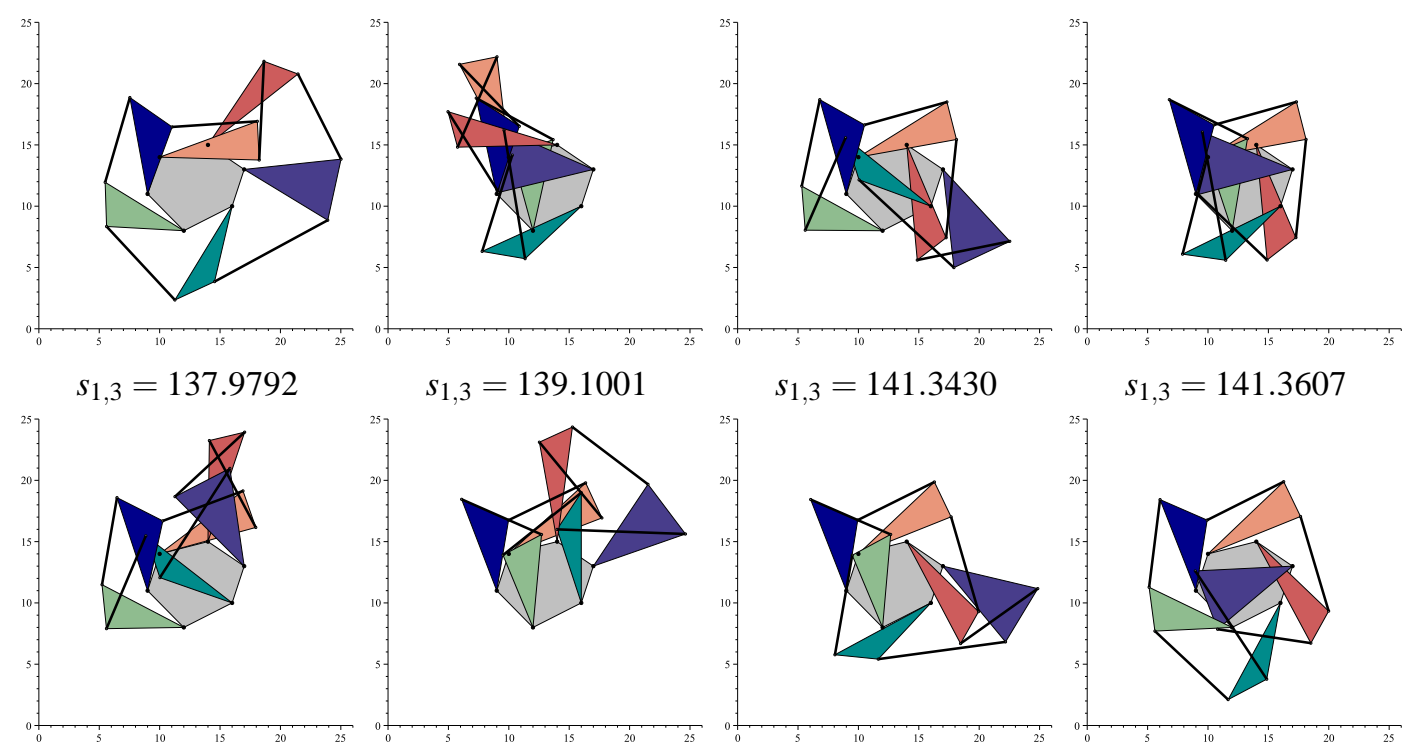

$s_{1,3}=139.1001$

$s_{1,3}=141.3430$

$s_{1,3}=141.3607$

$s_{1,3}=142.7141$
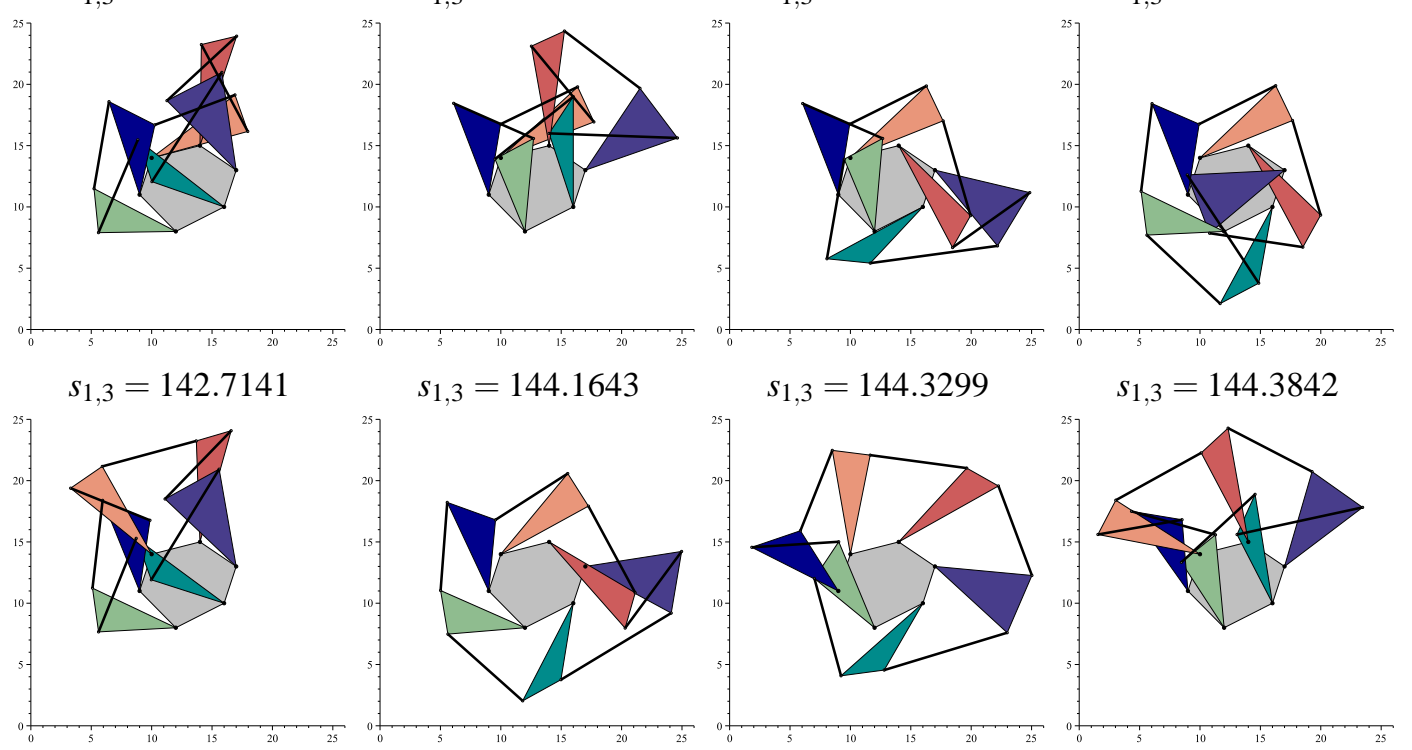

$s_{1,3}=144.1643$

$s_{1,3}=144.3299$

$s_{1,3}=144.3842$
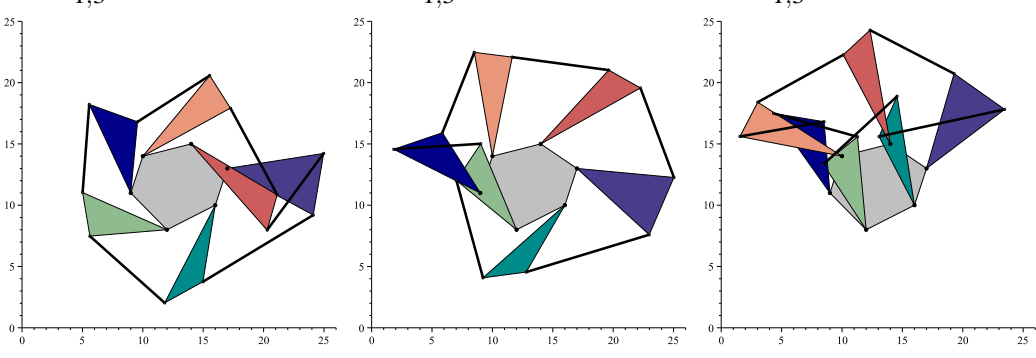

$s_{1,3}=144.7027$
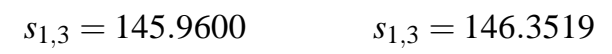

$s_{1,3}=148.9932$
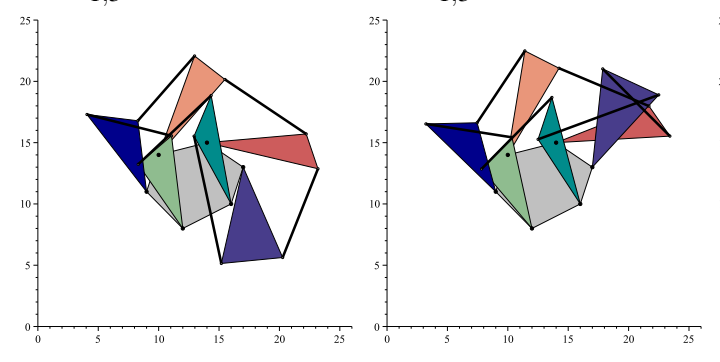

$s_{1,3}=149.3873$

$s_{1,3}=149.8649$

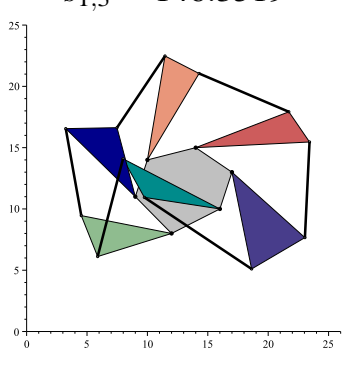

$s_{1,3}=149.8708$

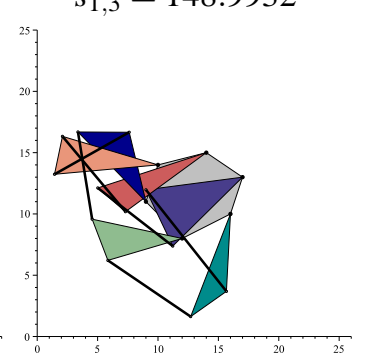

$s_{1,3}=149.8813$

Fig. 7. The assembly modes of the analyzed 13-link Watt-Baranov truss (Part 3)

pp. $112-126$.

[8] Burmester, L., 1888. Lehrbuch der Kinematik. Arthur Felix Verlag, Leipzig.

[9] Dhingra, A., Almadi, A., and Kohli, D., 2000. "A closed-form approach to coupler-curves of multi-loop mechanisms". Journal of Mechanical Design, 122(4), pp. 464-471.

[10] Thomas, F. Simulation of planar bar linkages using Cinderella. Web document available at http://www.iri.upc.edu/people/thomas/PlanarLinkages.html.

[11] Wohlhart, K., 1994. "Position analysis of the rhombic Assur group 4.4". In Proceedings of the RoManSy, CISM- IFTOMM Symposium, Gdansk, Poland, pp. 21 -31 .

[12] Lösch, S., 1995. "Parallel redundant manipulators based on open and closed normal Assur chains". In Computational Kinematics, J. Merlet and B. Ravani, eds. Kluver Academic Publishers, pp. 251-260.

[13] Wang, P., Liao, Q., Wei, S., and Zuang, Y., 2006. "Forward displacement analysis of a kind of nine-link Barranov truss based on Dixon resultants". China Mechanical Engineering, 17(21), pp. 2034 - 2038.

[14] Wang, P., Liao, Q., Zhuang, Y., and Wei, S., 2007. “A method for position analysis of a kind of nine-link Barranov truss". Mechanism and Machine Theory, 42(10), pp. $1280-1288$.

[15] Wohlhart, K., 2010. "Position analyses of normal quadrilateral Assur groups". Mechanism and Machine Theory, 45(9), pp. 1367 - 1384.

[16] Han, L., Liao, Q., and Liang, C., 1999. "A kind of algebraic solution for the position analysis of a planar basic 
kinematic chain". Journal of Machine Design, 16(3), pp. $16-18$.

[17] Borràs, J., and Gregorio, R. D., 2009. "Polynomial solution to the position analysis of two Assur kinematic chains with four loops and the same topology". Journal of Mechanisms and Robotics, 1(2), p. 021003.
[18] Wohlhart, K., 2009. "Position analyses of open normal Assur groups a(3.6)". In ASME/IFToMM International Conference on Reconfigurable Mechanisms and Robots, 2009. ReMAR 2009., pp. 88 -94.

[19] Blumenthal, L., 1953. Theory and applications of Distance Geometry. Oxford University Press. 